gr-qc/0308033

CGPG-03-07/3

NSF-KITP-03-57

\title{
Dynamical Horizons and their properties
}

\author{
Abhay Ashtekar ${ }^{1,2,4 *}$ and Badri Krishnan ${ }^{1,3,4 \dagger}$ \\ ${ }^{1}$ Center for Gravitational Physics and Geometry, \\ Physics Department, \\ Penn State University, \\ University Park, PA 16802, USA \\ ${ }^{2}$ Kavli Institute of Theoretical Physics \\ University of California, \\ Santa Barbara, CA 93106-4030, USA \\ ${ }^{3}$ Max Planck Institut für Gravitationsphysik \\ Albert Einstein Institut, \\ 14476 Golm, Germany \\ ${ }^{4}$ Erwin Schrödinger Institute, \\ Boltzmanngasse 9, 1090 Vienna, Austria
}

(Dated: October 31, 2018)

\begin{abstract}
A detailed description of how black holes grow in full, non-linear general relativity is presented. The starting point is the notion of dynamical horizons. Expressions of fluxes of energy and angular momentum carried by gravitational waves across these horizons are obtained. Fluxes are local and the energy flux is positive. Change in the horizon area is related to these fluxes. A notion of angular momentum and energy is associated with cross-sections of the horizon and balance equations, analogous to those obtained by Bondi and Sachs at null infinity, are derived. These in turn lead to generalizations of the first and second laws of black hole mechanics. The relation between dynamical horizons and their asymptotic states - the isolated horizons - is discussed briefly. The framework has potential applications to numerical, mathematical, astrophysical and quantum general relativity.
\end{abstract}

PACS numbers: 04.25.Dm, 04.70.Bw

*Electronic address: ashtekar@gravity.psu.edu

${ }^{\dagger}$ Electronic address: badkri@aei.mpg.de 


\section{INTRODUCTION}

Properties of stationary, 4-dimensional black holes have been well-understood for quite some time. In the Einstein-Maxwell theory, for example, the situation is astonishingly simple: We know that there is a unique 4-parameter family of stationary solutions and, furthermore, these solutions are known explicitly, in a closed form, given by the KerrNewman metrics and associated Maxwell fields [1]. Large families of stationary but distorted black holes are also known, where the distortion is caused by rings of matter and magnetic fields [2]. Finally, a framework has recently been introduced to probe properties of black holes which are themselves in equilibrium but in space-times with non-trivial dynamics in the exterior region [3-5]. In particular, this isolated horizon framework enables one to assign mass and angular momentum to black holes in terms of values of the fields on the horizon itself, without any reference to infinity and has also led to a generalization of the zeroth and first laws of black hole mechanics $[6,7]$.

However, in Nature, black holes are rarely in equilibrium. They grow by swallowing stars and galactic debris as well as electromagnetic and gravitational radiation. For such fully dynamical black holes, essentially there has been only one major result in exact general relativity. This is the celebrated area theorem, proved by Stephen Hawking in the early seventies $[8,9]$ : if matter satisfies the dominant energy condition, the area of the black hole event horizon can never decrease. This theorem has been extremely influential because of its similarity with the second law of thermodynamics. However, it is a qualitative result; it does not provide an explicit formula for the amount by which the area increases in physical situations. Now, the first law of black hole mechanics

$$
\delta E=(\kappa / 8 \pi G) \delta a+\Omega \delta J
$$

does relate the change in the area of an isolated horizon to that in the energy and angular momentum, as the black hole makes a transition from one equilibrium state to a nearby one. This suggests that there may well be a fully dynamical version of (1.1) which relates the change in the black hole area to the energy and angular momentum fluxes, as the black hole makes a transition from a given state to one which is far removed. Thus, we are naturally led to ask: Can the results obtained in the isolated horizon framework be extended to fully dynamical situations?

Attractive as this possibility seems, one immediately encounters a serious conceptual and technical problem. For, the expression requires, in particular, a precise notion of the flux of gravitational energy across the horizon. Already at null infinity, the expression of the gravitational energy flux is subtle: one needs the framework developed by Bondi, Sachs, Newman, Penrose and others to introduce a viable, gauge invariant expression of this flux [10-12]. In the strong field regime, there is no satisfactory generalization of this framework and no satisfactory, gauge invariant notion of gravitational energy flux beyond perturbation theory. Thus, one appears to be stuck right at the start.

Yet, there are at least two general considerations that suggest that an extension of the first law to fully dynamical situations should be possible. Consider a stellar collapse leading to the formation of a black hole. At the end of the process, one has a black hole and, from general physical considerations, one expects that the energy in the final black hole should equal the total matter plus gravitational energy that fell across the horizon. Thus, at least the total integrated flux across the horizon should be well defined. Indeed, it should equal the depletion of the energy in the asymptotic region, i.e., the difference between the 
ADM energy and the energy radiated across future null infinity. The second consideration involves the Penrose inequalities which were motivated by cosmic censorship: The ADM mass should be greater than or equal to the half the radius of the apparent horizon on any (partial) Cauchy slice [13]. (Special cases of this conjecture have been proved recently [14].) Heuristically, the inequality leads us to think of the apparent horizon radius as a measure of the mass in its interior, whence one is led to conclude that the change in the area is due to influx of energy. Thus, it is tempting to hope that something special may happen at the surface of a black hole enabling one to define the flux of energy and angular momentum across it, thereby giving a precise meaning to these physical expectations.

The question then is: how should we define the surface of the black hole? The obvious candidate is the event horizon. Unfortunately, this is not a viable possibility because event horizons are extremely global and teleological. Consider for example the gravitational collapse of a thin spherical shell. The event horizon first forms in the interior of the shell and then expands out. Thus, in the initial phase, it lies in a flat space-time region and expands out in anticipation that the shell will cross it, even though neither the matter nor the gravitational radiation falls across it before it hits the shell. Thus, one cannot hope to find a quasi-local, fully dynamical generalization of the first law using event horizons. However, there is an alternative, suggested by the strategy used routinely in numerical simulations of black hole formation or coalescence. There, one avoids the problems associated with the global and teleological nature of the event horizon by locating apparent horizons at each time during evolution. ${ }^{1}$ Can one then use apparent horizons to obtain the desired generalization of eq. (1.1)? Now, apparent horizons can and do jump during evolution. However, in all numerical simulations, there are epochs during which the world tube $\tau_{\mathrm{AH}}$ traced out by apparent horizons is smooth. The rough, intuitive idea is to use these world tubes as the black hole surfaces across which energy and angular momentum fluxes are to be calculated.

We will incorporate these heuristics in a precise notion called dynamical horizons. However, the definition will only involve conditions on a 3-surface $H$, extracted from the expected properties of $\tau_{\mathrm{AH}}$. In particular, the definition will not make any reference to space-time foliations and apparent horizons thereon. Indeed, the definition will be quasi-local. Thus, given a region of space-time, one can tell whether or not it admits dynamical horizons, without any knowledge of the geometry and matter fields in the exterior region. Similarly, given a specific 3-dimensional sub-manifold, one can decide whether it is a dynamical horizon by examining space-time fields defined on it, without the knowledge of geometry and matter fields away from the surface. By construction, the world tubes $\tau_{\mathrm{AH}}$ will provide examples of dynamical horizons which are most useful to numerical relativity. However, using Hayward's [15] notion of trapping boundaries, one can also associate with a generic evolving black hole a more invariantly defined or canonical dynamical horizon. From a general conceptual viewpoint, it may seem more natural to restrict oneself just to these canonical dynamical horizons. However, for 'practical' applications, this would be too restrictive. For, although these horizons do not refer to global notions such as null infinity, they are nonetheless difficult to locate in a given space-time. A key strength of the approach is that our analysis is not tied just to them but encompasses all dynamical horizons. In particular, we will be

\footnotetext{
${ }^{1}$ In this paper, the term 'apparent horizon' is used in the sense employed in snumerical relativity: it is the outermost marginally trapped surface on a given (partial) Cauchy slice. By contrast, Hawking and Ellis [9] define an apparent horizon as the boundary of a trapped region associated with the Cauchy slice (i.e., of a connected region through each point of which there passes an outer trapped surface lying in the slice.
} 
able to introduce flux formulas and an integral generalization of the first law (1.1) which will hold on all dynamical horizons, including the ones of interest to numerical relativity.

The paper is organized as follows. In section II, we introduce the main definitions, motivate the conditions and explain the relation to Hayward's trapping horizons. In section III we derive an area balance law, relating the change in the area of the dynamical horizon to the flux of matter energy and a pure geometrical, positive definite term. We then interpret the geometrical term as the flux of gravitational energy and show that it satisfies the criteria one normally uses to establish the viability of the Bondi flux formula at null infinity. Section IV introduces the notion of angular momentum and section $\mathrm{V}$ extends the area balance law using angular momentum considerations to an integral form of the first law. Using strategies that have been successful in the isolated horizon framework, we also introduce a definition of horizon energy and show that it matches well with the flux formulas to provide an energy balance law analogous to that at null infinity, but now in the strong field regime of dynamical horizons. While the horizon would be dynamical in the time dependent phase of black hole formation or soon after two black holes merge, one expects it to settle down and reach equilibrium at late times. Thus, one would expect isolated horizons to be the asymptotic states of dynamical horizons. In section VI we explore the relation between the two. Section VII summarizes the overall situation, suggests applications of dynamical horizons to numerical, mathematical and quantum relativity and lists problems in these areas whose resolution would shed much new light on how black holes grow and settle down to their final states.

To preserve the flow of discussion in the main paper, some issues have been postponed to appendices. Appendix A discusses the simplest explicit examples of dynamical horizons and their passage to equilibrium. For completeness, in Appendix B we discuss the time-like analogs of dynamical horizons which arise in cosmological contexts.

The main results of this work were briefly reported in $[16,17]$. Here we present the details, proofs and extensions of those results.

\section{DEFINITIONS AND THE METHOD}

In this section, we will introduce the basic definitions, explain in some detail the motivation behind them, discuss the relation between dynamical horizons and closely related notions of trapping horizons introduced by Hayward [15], and outline the main idea on which calculations in the subsequent sections are based.

\section{A. Definition and motivation}

Definition 1: A smooth, three-dimensional, space-like sub-manifold $H$ in a space-time $\mathcal{M}$ is said to be a dynamical horizon if it can be foliated by a family of closed 2-surfaces such that, on each leaf $S$, the expansion $\Theta_{(\ell)}$ of one null normal $\ell^{a}$ vanishes and the expansion $\Theta_{(n)}$ of the other null normal $n^{a}$ is strictly negative. ${ }^{2}$

\footnotetext{
2 This notion of dynamical horizons is slightly more general than that used in the brief reports $[16,17]$
} where the foliation was fixed and the topology of the leaves of the foliation was required to be $S^{2}$. 
Thus, basically a dynamical horizon $H$ is a space-like 3-manifold which is foliated by closed, marginally trapped 2-surfaces. Note first that, in contrast to event horizons, dynamical horizons can be located quasi-locally; knowledge of full space-time is not required. Thus, for example, while an event horizon may well be developing in the room in which you are now sitting in anticipation of a future gravitational collapse, you can rest assured that no dynamical horizon has ever developed in that room! Next, since event horizons are defined as the future boundary of the causal past of future null infinity, the notion is tied to asymptotically flat space-times. Being quasi-local, the notion of dynamical horizons does not refer to the asymptotic structure at all and is meaningful also in spatially compact space-times. On the other hand, while in asymptotically flat space-times black holes are characterized by event horizons, there is no one-to-one correspondence between black holes and dynamical horizons. First of all, we expect that stationary black holes do not admit dynamical horizons because these space-times are non-dynamical. In time dependent situations, if the dominant energy condition holds and the space-time is asymptotically predictable, dynamical horizons lie inside the event horizon. However, in the interior of an expanding event horizon, there may be many dynamical horizons. Nonetheless, in the sense made precise in section IIB, under fairly general conditions one can associate with each evolving black hole an outermost or canonical dynamical horizon. For conceptual reasons, it is natural to focus just on this canonical one. However, our results will apply to all dynamical horizons; indeed, it is this fact that makes the framework powerful in practice, e.g., for applications to numerical relativity.

Apart from the requirement that $H$ be foliated by marginally trapped surfaces, the definition contains three conditions. The first asks that the 2 -surfaces which constitute the leaves of the foliation be closed. This condition is necessary to ensure the convergence of various integrals we will perform. The second asks that the expansion $\Theta_{(n)}$ be strictly negative. This condition is quite weak because, in essence, it simply enables one to identify $n^{a}$ as the inward pointing null normal. Thus, had $\Theta_{(n)}$ been positive, we would be in the white hole situation, rather than the black hole one. Nonetheless, the condition is restrictive in a minor way: it rules out the degenerate case in which $\Theta_{(n)}$ vanishes. As we will show below, the area of the trapped surfaces increases if $\Theta_{(n)}$ is negative and remains constant if it vanishes. Thus, by removing the degenerate case, we are basically ignoring the non-dynamical situation. One might consider intermediate dynamical situations in which $\Theta_{(n)}$ vanishes on a portion of each marginally trapped surface and is negative elsewhere. In this case, the total area would still increase. Our main results will continue to be valid in these intermediate cases.

The third condition is that $H$ be space-like. Intuitively, it is clear that if $H$ were timelike, it would not be a boundary of a black hole region because light rays originating on $H$ would propagate on both sides of the space-time separated by $H$. So, the non-triviality lies in the fact that this condition rules out the possibility that $H$ could be null. To probe how much of a restriction this is physically, let us proceed by dropping the requirement that $H$ be space-like but keeping the other conditions in Definition 1 . Denote by $V^{a}$ a vector field which is tangential to $H$, everywhere orthogonal to the foliation by marginally trapped surfaces and preserves this foliation. We can always choose the normalization of $\ell^{a}$ and $n^{a}$ such that $\ell^{a} n_{a}=-2$ and $V^{a}=\ell^{a}-f n^{a}$ for some $f$. Since $V \cdot V=4 f$, it follows that $H$ is respectively, space-like, null or time-like, depending on whether $f$ is positive, zero or negative. We will argue that under conditions that capture the physics we have in mind, generically $f$ would be non-negative. Let us begin by noting that the definition of $V^{a}$ immediately implies $\mathcal{L}_{V} \Theta_{(\ell)}=0$, whence, $\mathcal{L}_{\ell} \Theta_{(\ell)}=f \mathcal{L}_{n} \Theta_{(\ell)}$. Therefore, the Raychaudhuri 
equation for $\ell^{a}$ implies

$$
f \mathcal{L}_{n} \Theta_{(\ell)}=-\sigma^{2}-R_{a b} \ell^{a} \ell^{b}
$$

where $\sigma$ is the shear of $\ell^{a}$. Now, given the scenario we have in mind, it is physically reasonable to assume that the convergence $\Theta_{(\ell)}$ of $\ell^{a}$ becomes negative as one moves along $n^{a}$ to the interior of the marginally trapped surfaces, whence $\mathcal{L}_{n} \Theta_{(\ell)}<0$. If matter satisfies the dominant energy condition, the right side of (2.1) is non-positive, whence we conclude that $f$ is non-negative; as expected the time-like case is ruled out. Finally, as we will show in section III, if the flux of energy across $H$ is non-zero on any one leaf of the foliation of $H$, the right side of (2.1) cannot vanish identically on that leaf. Thus, under the intended dynamical situations, $f$ would be strictly positive somewhere on each leaf, whence $H$ would be space-like there. By requiring that $H$ be space-like everywhere we are ignoring the case in which portions of marginally trapped surfaces lie on a space-like horizon and the remainder on a null horizon. This case will be discussed elsewhere [18] but we will comment on how some of the main results are modified in this case. Finally, the assumption that $H$ is spacelike also rules out situations in which the horizon reaches equilibrium and the energy flux across entire cross-sections vanishes. These will be considered in section VI.

To summarize, apart from the possibility that $H$ may be partially null as discussed separately in section VI and in [18], for evolving black holes the conditions imposed in Definition 1 are natural and incorporate most of the physical situations we have in mind. The world-tubes $\tau_{\mathrm{AH}}$ of apparent horizons resulting from 'nice' foliations of numerically simulated space-times will probably satisfy our conditions and qualify as dynamical horizons. (For random foliations, the intuitive condition $\mathcal{L}_{n} \Theta_{(\ell)}<0$ may be violated, whence $\tau_{\text {AH }}$ may well be partially time-like.) But the notion of dynamical horizons appears to be more general in the sense that we do not know of a result to the effect that given a dynamical horizon $H$, the space-time must admit a foliation for which cross-sections $S$ of $H$ are apparent horizons (rather than just marginally trapped surfaces, which they certainly are). Finally, explicit examples of dynamical horizons are provided by the Vaidya metrics discussed in some detail in Appendix A. (In this case, the topology of the cross-sections $S$ is $S^{2}$ and the generic condition $\mathcal{L}_{n} \Theta_{(\ell)}<0$ is satisfied in the dynamical black hole region.) Thus, overall, the requirements in the Definitions are rather mild. In the remainder of this paper we will see that the conditions are also sufficiently strong in the sense that the Definition has a rich variety of consequences.

\section{B. Hayward's trapping horizons}

To capture the notion of a black hole without reference to infinity, Hayward [15] constructed an ingenious quasi-local framework. Dynamical horizons are closely related to his notion of trapping horizons. In this subsection, we will clarify the relation between the two. This discussion will be especially useful to section VI because trapping horizons provide a natural arena for analyzing the transition at late times from dynamical to isolated horizons. Definition 2: A future, outer, trapping horizon (FOTH) is a 3-manifold, $H^{\prime}$, foliated by closed surfaces $S^{\prime}$ such that: i) the expansion of one future directed null normal $\ell^{a}$ to the foliation vanishes, $\Theta_{(\ell)}=0$; ii) the expansion of the other future directed null normal, $n^{a}$ is negative, $\Theta_{(n)}<0$; iii) the directional derivative of $\Theta_{(\ell)}$ along $n^{a}$ is negative; $\mathcal{L}_{n} \Theta_{(\ell)}<0$.

Here, condition ii) captures the idea that $H^{\prime}$ is a future horizon (i.e., of the black hole rather than white hole type) and condition iii) encodes the idea that it is 'outer' and serves 
to distinguish black hole type horizons from certain cosmological ones [15] which are not ruled out by condition ii).

Our discussion of section II B shows that $H^{\prime}$ is either space-like or null, being null if and only if the shear $\sigma$ of $\ell^{a}$ as well as the matter flux $T_{a b} \ell^{a} \ell^{b}$ across $H$ vanishes. A spacelike FOTH is a dynamical horizon on which the additional condition $\mathcal{L}_{n} \Theta_{(\ell)}<0$ holds. Similarly, a dynamical horizon satisfying $\mathcal{L}_{n} \Theta_{(\ell)}<0$ is a space-like FOTH. Thus, while neither Definition implies the other, there is a large overlap between dynamical horizons and FOTHs. In generic dynamic situations pertaining to black holes, one is likely to encounter horizons which satisfy both sets of conditions, i.e., lie in the intersection of the two sets. In fact, since one expects the region to the immediate future of the dynamical horizon to be trapped, a stronger version of $\mathcal{L}_{n} \Theta_{(\ell)}<0$ should be satisfied: if $\widehat{\tau}^{a}$ is a future directed normal to $H$ and $W^{a}$ is any vector such that $W^{a} \widehat{\tau}_{a}<0$, then $\mathcal{L}_{W} \Theta_{(\ell)}<0$.

The advantage of Definition 1 is that it refers only to the intrinsic structure of $H$, without any conditions on the evolution of fields in directions transverse to $H$. As we will see, this makes it natural to analyze the structure of $H$ using only the constraint (or initial value) equations. Reciprocally, Definition 2 has the advantage that it permits $H^{\prime}$ to be space-like or null. In a spherical collapse of a scalar field, for example, while $H$ is useful only in the regions where the flux of the scalar field energy across $H^{\prime}$ is non-zero, $H^{\prime}$ is useful also in the region where it vanishes and the horizon becomes null. (See section VI and the explicit examples discussed in Appendix A.)

Finally, we recall Hayward's [15] notions related to a trapping boundary. A trapped region is a connected subset of space-time through each point $p$ of which there passes a closed trapped surface (such that $\Theta_{(\ell)}<0$ and $\Theta_{(n)}<0$ ). An inextendable trapped region $\mathbf{T}$ is a trapped region that cannot be extended. A trapping boundary $\partial \mathbf{T}$ is the boundary of an inextendable trapped region $\mathbf{T}$. Physically, $\mathbf{T}$ can be regarded as a black hole region of the space-time and $\partial \mathbf{T}$, as the surface of that black hole. To establish a desired property of this surface, Hayward had to introduce a further technical notion: A limit section of the trapping boundary is a smooth, closed sub-manifold of $\partial \mathbf{T}$ which can be obtained as an uniform limit of closed trapped surfaces lying in $\mathbf{T}$. With these definitions at hand, Hayward showed that if a trapping boundary is smooth and foliated by limit sections, then the following conditions hold on each leaf : i) The expansion of one of the null normal, say $\ell^{a}$ vanishes; $\Theta_{(\ell)}=0$; ii) The expansion of the second null normal satisfies $\Theta_{(n)} \leq 0$; and iii) $\mathcal{L}_{n} \Theta_{(\ell)} \leq 0$. Thus, if we ignore the degenerate cases where equalities hold in the last two equations, the boundary is a FOTH. In this sense then, generically, if the black hole is genuinely dynamical, the trapping boundary $\partial \mathbf{T}$ would be a dynamical horizon, and if it has reached equilibrium, it would be a weakly isolated horizon [6]. In the former case, $\partial \mathbf{T}$ would represent the canonical dynamical horizon associated with the black hole under consideration.

\section{Notation and strategy}

In the next four sections of this paper, we will consider a dynamical horizon $H$ and explore its properties. If $H$ admits more than one foliation by marginally trapped surfaces satisfying Definition 1, we will just choose any one of them and use it throughout our calculations. Our results will apply to all such foliations. At appropriate places, we will comment on the expressions which are foliation independent. Leaves of the fixed foliation will be called cross-sections of $H$. 
Let us begin by specifying notation. For simplicity, All manifolds will be assumed to be smooth (i.e. $C^{k+1}$ with $k \geq 3$ ) and orientable and all fields will be assumed to be smooth (i.e., $\left.C^{k}\right)$. The space-time metric $g_{a b}$ has signature $(-,+,+,+)$ and its derivative operator will be denoted by $\nabla$. The Riemann tensor is defined by $R_{a b c}{ }^{d} W_{d}:=2 \nabla_{[a} \nabla_{b]} W_{c}$, the Ricci tensor by $R_{a b}:=R_{a c b}{ }^{c}$ and the scalar curvature by $R:=g^{a b} R_{a b}$. We will assume the field equations

$$
R_{a b}-\frac{1}{2} R g_{a b}+\Lambda g_{a b}=8 \pi G T_{a b}
$$

(With these conventions, de Sitter space-time has positive cosmological constant $\Lambda$.) We assume that $T_{a b}$ satisfies the dominant energy condition (although, as the reader can easily tell, several of the results will hold under weaker restrictions.) To keep the discussion reasonably focussed, we will not consider gauge fields with non-zero charges on the horizon. Inclusion of these fields is not difficult but introduces a number of subtleties and complications which are irrelevant for numerical relativity and astrophysics. They will be discussed elsewhere.

Geometry of the dynamical horizon $H$ is pictorially represented in figure 1 . The unit normal to $H$ will be denoted by $\widehat{\tau}^{a} ; g_{a b} \widehat{\tau}^{a} \widehat{\tau}^{b}=-1$. The intrinsic metric and the extrinsic curvature of $H$ are denoted by $q_{a b}:=g_{a b}+\widehat{\tau}_{a} \widehat{\tau}_{b}$ and $K_{a b}:=q_{a}{ }^{c} q_{b}{ }^{d} \nabla_{c} \widehat{\tau}_{d}$ respectively. $D$ is the derivative operator on $H$ compatible with $q_{a b}, \mathcal{R}_{a b}$ its Ricci tensor and $\mathcal{R}$ its scalar curvature. The unit space-like vector orthogonal to $S$ and tangent to $H$ is denoted by $\widehat{r}^{a}$. Quantities intrinsic to $S$ will be generally written with a tilde. Thus, the two-metric on $S$ is $\widetilde{q}_{a b}$ and the extrinsic curvature of $S \subset H$ is $\widetilde{K}_{a b}:=\widetilde{q}_{a}{ }^{c} \widetilde{q}_{b}{ }^{d} D_{c} \widehat{r}_{d}$; the derivative operator on $\left(S, \widetilde{q}_{a b}\right)$ is $\widetilde{D}$ and its Ricci tensor is $\widetilde{\mathcal{R}}_{a b}$. Finally, in the next four sections we will fix the rescaling freedom in the choice of null normals via $\ell^{a}:=\widehat{\tau}^{a}+\widehat{r}^{a}$ and $n^{a}:=\widehat{\tau}^{a}-\widehat{r}^{a}$ (so that $\left.\ell^{a} n_{a}=-2\right)$. This convention will have to be modified in the discussion of transition to equilibrium of section VI.

We first note an immediate consequence of the definition. Since $\Theta_{(\ell)}=0$ and $\Theta_{(n)}<0$, it follows that

$$
\widetilde{K}=\tilde{q}^{a b} D_{a} \widehat{r}_{b}=\frac{1}{2} \tilde{q}^{a b} \nabla_{a}\left(\ell_{b}-n_{b}\right)=-\frac{1}{2} \Theta_{(n)}>0 .
$$

Hence the area $a_{S}$ of $S$ increases monotonically along $\widehat{r}^{a}$. Thus the second law of black hole mechanics holds on $H$. Our first task is to obtain an explicit expression for the change of area.

Our main analysis is based on the fact that, since $H$ is a space-like surface, the Cauchy data $\left(q_{a b}, K_{a b}\right)$ on $H$ must satisfy the usual scalar and vector constraints

$$
\begin{aligned}
& H_{S}:=\mathcal{R}+K^{2}-K^{a b} K_{a b}=16 \pi G \bar{T}_{a b} \widehat{\tau}^{a} \widehat{\tau}^{b} \\
& H_{V}^{a}:=D_{b}\left(K^{a b}-K q^{a b}\right)=8 \pi G \bar{T}^{b c} \widehat{\tau}_{c} q_{b}^{a} .
\end{aligned}
$$

where

$$
\bar{T}_{a b}=T_{a b}-\frac{1}{8 \pi G} \Lambda g_{a b}
$$

and $T_{a b}$ is the matter stress-energy tensor. The strategy behind the key calculations in the next three sections is entirely straightforward: We will fix two cross-sections $S_{1}$ and $S_{2}$ of $H$, multiply $H_{S}$ and $H_{V}^{a}$ with appropriate lapse and shift fields and integrate the result on a portion $\Delta H \subset H$ which is bounded by $S_{1}$ and $S_{2}$.

Remark: As noted in section II B, the notions of dynamical horizons and FOTHs are closely related and, in physically interesting situations involving evolving black holes, both sets of conditions will be satisfied. However, there are key differences between our analysis 


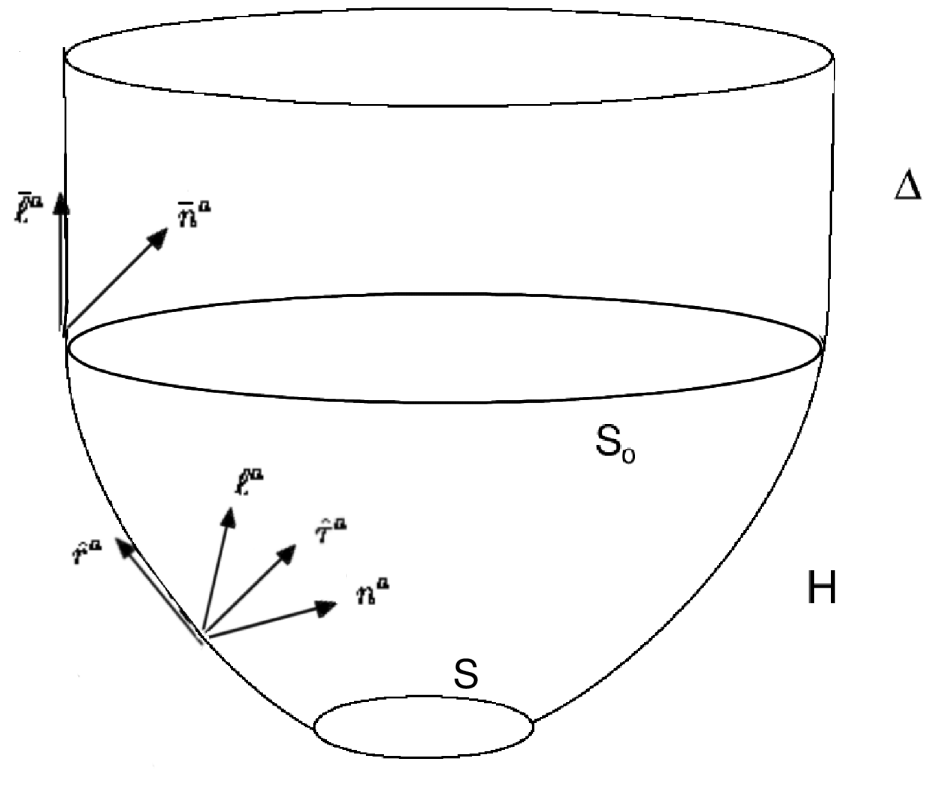

FIG. 1: $H$ is a dynamical horizon, foliated by marginally trapped surfaces $S . \widehat{\tau}^{a}$ is the unit time-like normal to $H$ and $\widehat{r}^{a}$ the unit space-like normal within $H$ to the foliations. Although $H$ is space-like, motions along $\widehat{r}^{a}$ can be regarded as time evolution with respect to observers at infinity. In this respect, one can think of $H$ as a hyperboloid in Minkowski space and $S$ as the intersection of the hyperboloid with space-like planes. $H$ joins on to a weakly isolated horizon $\Delta$ with null normal $\bar{\ell}^{a}$, at a cross-section $S_{o}$.

based on dynamical horizons and Hayward's analysis [15] based on FOTHs . While our analysis will be based on the standard 3+1 decomposition, Hayward's framework is based on a $2+2$ decomposition. The $2+2$ framework is better suited for analyzing more general horizons where $H$ is partially time-like and partially null but has the disadvantage that it fails to make it manifestly clear that the fields of interest are defined just by the horizon geometry and are independent of extensions used off $H$. In terms of results, our final result on the topology of cross-sections is the same as that of [15]. However, results in the rest of the paper are quite different. Specifically, our flux formulae are new, our discussion includes angular momentum, our generalization of black hole mechanics is different, and our definition of the horizon energy and balance laws are new. 


\section{ENERGY FLUXES AND AREA BALANCE}

Let us now turn to the task of relating the change in area to the flux of energy across $H$. Along the way, we will establish that the topology of the cross sections $S$ of $H$ is severely restricted in the case when $\Lambda \geq 0$.

\section{A. Area increase and topology of $S$}

As is usual in general relativity, the notion of energy is tied to a choice of a vector field. The definition of a dynamical horizon provides a preferred direction field; that along $\ell^{a}$. To fix the proportionality factor, or the lapse $N$, let us first introduce the area radius $R$, a function which is constant on each $S$ and satisfies $a_{S}=4 \pi R^{2}$. Since we already know that area is monotonically increasing, $R$ is a good coordinate on $H$. Now, the 3 -volume $d^{3} V$ on $H$ can be decomposed as $d^{3} V=|\partial R|^{-1} d R d^{2} V$ where $\partial$ denotes the gradient on $H$. Therefore, as we will see, our calculations will simplify if we choose $N_{R}=|\partial R|$. In this sub-section, we will make this simple choice, obtain an expression for the change in area and show that the topology of the cross-sections $S$ is severely restricted. In section III C we will generalize this area balance law to include a more general family of lapses.

Since the area increase formula plays an important role throughout the paper, we will provide a detailed derivation. Fix two cross sections $S_{1}$ and $S_{2}$ of $H$ and denote by $\Delta H$ the portion of $H$ they bound. We are interested in calculating the flux of energy associated with $\xi_{(R)}^{a}=N_{R} \ell^{a}$ across $\Delta H$. Denote the flux of matter energy across $\Delta H$ by $\mathcal{F}_{\text {matter }}^{(R)}$ :

$$
\mathcal{F}_{\text {matter }}^{(R)}:=\int_{\Delta H} T_{a b} \widehat{\tau}^{a} \xi_{(R)}^{b} d^{3} V
$$

By taking the appropriate combination of (2.4) and (2.5) we obtain

$$
\begin{aligned}
\mathcal{F}_{\text {matter }}^{(R)} & =\frac{1}{16 \pi G} \int_{\Delta H} N_{R}\left(H_{S}+2 \widehat{r}_{a} H_{V}^{a}\right) d^{3} V \\
& =\frac{1}{16 \pi G} \int_{\Delta H} N_{R}\left(\mathcal{R}+K^{2}-K^{a b} K_{a b}+2 \widehat{r}_{a} D_{b} P^{a b}\right) d^{3} V
\end{aligned}
$$

where $P^{a b}$ is defined as

$$
P^{a b}=K^{a b}-K q^{a b} .
$$

Since $H$ is foliated by compact 2 -manifolds $S$, we can perform a $2+1$ decomposition of various quantities on $H$. First, the Gauss-Codacci equation relating the space-time curvature to the intrinsic curvature of $H$ leads to

$$
2 \mathcal{G}_{a b} \widehat{r}^{a} \widehat{r}^{b}=-\widetilde{\mathcal{R}}+\widetilde{K}^{2}-\widetilde{K}_{a b} \widetilde{K}^{a b}
$$

where $\mathcal{G}_{a b}$ is the Einstein tensor of $\left(H, q_{a b}\right)$, and the definition of the Riemann tensor gives

$$
\mathcal{R}_{a b} \widehat{r}^{a} \widehat{r}^{b}=-2 \widehat{r}^{a} D_{[a} D_{b]} \widehat{r}^{b}=D_{a} \alpha^{a}+\widetilde{K}^{2}-\widetilde{K}_{a b} \widetilde{K}^{a b}
$$

where

$$
\alpha^{a}:=\widehat{r}^{b} D_{b} \widehat{r}^{a}-\widehat{r}^{a} D_{b} \widehat{r}^{b} .
$$


Combining equations (3.4) and (3.5), we can obtain a useful expression relating the scalar curvatures on $H$ and $S$ :

$$
\mathcal{R}=2\left(\mathcal{R}_{a b}-\mathcal{G}_{a b}\right) \widehat{r}^{a} \widehat{r}^{b}=\widetilde{\mathcal{R}}+\widetilde{K}^{2}-\widetilde{K}_{a b} \widetilde{K}^{a b}+2 D_{a} \alpha^{a}
$$

Transvecting the momentum constraint equation with $\widehat{r}_{b}$ gives

$$
\widehat{r}_{b} D_{a} P^{a b}=D_{a} \beta^{a}-P^{a b} D_{a} \widehat{r}_{b}
$$

where

$$
\beta^{a}:=K^{a b} \widehat{r}_{b}-K \widehat{r}^{a} .
$$

Substituting the results of equations (3.7) and (3.8) into the integrand of the right side of eq. (3.2) yields

$$
H_{S}+2 \widehat{r}_{a} H_{V}^{a}=\widetilde{\mathcal{R}}+\widetilde{K}^{2}-\widetilde{K}_{a b} \widetilde{K}^{a b}+K^{2}-K_{a b} K^{a b}-2 P^{a b} D_{a} \widehat{r}_{b}+2 D_{a} \gamma^{a}
$$

where

$$
\gamma^{a}:=\alpha^{a}+\beta^{a}
$$

For further simplification, let us bear in mind that we will eventually use the key property that the cross sections $S$ are marginally trapped surfaces, i. e. $\Theta_{(\ell)}=0$. In terms of the extrinsic curvatures $K_{a b}$ and $\widetilde{K}_{a b}$, the expansion can be written as

$$
\Theta_{(\ell)}=K-K_{a b} \widehat{r}^{a} \widehat{r}^{b}+\widetilde{K}
$$

To recast the extrinsic curvature terms in Eq. (3.10) using $\Theta_{(\ell)}$, it is convenient to perform a decomposition of the two extrinsic curvatures:

$$
\begin{aligned}
\widetilde{K}_{a b} & =\frac{1}{2} \widetilde{K} \widetilde{q}_{a b}+\widetilde{S}_{a b} \\
K_{a b} & =A \widetilde{q}_{a b}+S_{a b}+2 \widetilde{W}_{(a} \widehat{r}_{b)}+B \widehat{r}_{a} \widehat{r}_{b},
\end{aligned}
$$

where $\widetilde{S}_{a b}$ is the trace-free part of $\widetilde{K}_{a b} ; S_{a b}$, the trace-free part of the projection of $K_{a b}$ into $S ; \widetilde{W}_{a}$ is the projection of $K_{a b} \widehat{r}^{b}$ into $S ; A:=\frac{1}{2} K_{a b} \widetilde{q}^{a b}$ and $B:=K_{a b} \widehat{r}^{a} \widehat{r}^{b}$. Note that $S_{a b}, \widetilde{S}_{a b}$ and $\widetilde{W}_{a}$ are two-dimensional tensors intrinsic to the cross-section $S$. Substituting the above decompositions in eq. (3.10) $\mathcal{F}_{\text {matter }}^{(R)}$ and using eq. (3.12), we obtain

$$
\begin{aligned}
H_{S}+2 \widehat{r}_{a} H_{V}^{a}= & \widetilde{\mathcal{R}}-\sigma_{a b} \sigma^{a b}-2 \widetilde{W_{a}} \widetilde{W^{a}}-2 \widetilde{W^{a}} \widehat{r}^{b} D_{b} \widehat{r}_{a} \\
& +\frac{1}{2} \Theta_{(\ell)}\left(\Theta_{(\ell)}+4 B\right)+2 D_{a} \gamma^{a}
\end{aligned}
$$

where $\sigma_{a b}=S_{a b}+\widetilde{S}_{a b}$ is the shear of the null vector $\ell^{a}=\widehat{\tau}^{a}+\widehat{r}^{a}$; i.e. $\sigma_{a b}:=\widetilde{q}_{a}{ }^{m} \widetilde{q}_{b}{ }^{n} \nabla_{m} \ell_{n}-$ $\frac{1}{2} \widetilde{q}_{a b} \widetilde{q}^{m n} \nabla_{m} \ell_{n}$. Our task in the remainder of this calculation is to simplify the right side of this equation.

With this goal in mind, let us now turn our attention to the vector $\gamma^{a}$ defined in eqs. (3.11), (3.6), and (3.9):

$$
\begin{aligned}
\gamma^{a}=\alpha^{a}+\beta^{a} & =\widehat{r}^{b} D_{b} \widehat{r}^{a}-\widehat{r}^{a} D_{b} \widehat{r}^{b}+K^{a b} \widehat{r}_{b}-K \widehat{r}_{a} \\
& =\widehat{r}^{b} D_{b} \widehat{r}^{a}+\widetilde{W}^{a}-\Theta_{(\ell)} \widehat{r}^{a} .
\end{aligned}
$$


Finally, it is convenient to re-express the acceleration term as

$$
\widehat{r}^{a} D_{a} \widehat{r}_{b}=\left(N_{R}\right)^{-1} \widetilde{D}_{b} N_{R} .
$$

Then, Eq. (3.15) can be rewritten as:

$$
\begin{aligned}
H_{S}+2 \widehat{r}_{a} H_{V}^{a} & =\widetilde{\mathcal{R}}-\sigma_{a b} \sigma^{a b}-2 \zeta^{a} \zeta_{a}+2 \widetilde{D}_{a} \zeta^{a} \\
& +\frac{1}{2} \Theta_{(\ell)}\left(4 K-3 \Theta_{\ell)}\right)-2 \widehat{r}^{a} D_{a} \Theta_{(\ell)}
\end{aligned}
$$

where the vector $\zeta^{a}$, tangent to the cross sections, is defined as

$$
\zeta^{a}:=\widetilde{W}^{a}+\widetilde{D}^{a} \ln N_{R}=\widetilde{q}^{a b} \widehat{r}^{c} \nabla_{c} \ell_{b} .
$$

Equation (3.18) is completely general; it holds on any foliated space-like surface. We now wish to use the fact that surface of interest is in fact a dynamical horizon. Integrating on the portion $\Delta H$ of the horizon $H$, using the fact that the cross-sections $S$ are compact and $\Theta_{(\ell)}$ vanishes, we are led to a remarkably simple result:

$$
\mathcal{F}_{\text {matter }}^{(R)}=\frac{1}{16 \pi G} \int_{\Delta H} N_{R}\left(\widetilde{\mathcal{R}}-\sigma_{a b} \sigma^{a b}-2 \zeta^{a} \zeta_{a}\right) d^{3} V
$$

Using the abbreviations $|\sigma|^{2}:=\sigma_{a b} \sigma^{a b}$ and $|\zeta|^{2}:=\zeta_{a} \zeta^{a}$, this can be rewritten as

$$
\int_{\Delta H} N_{R} \widetilde{\mathcal{R}} d^{3} V=16 \pi G \int_{\Delta H} \bar{T}_{a b} \widehat{\tau}^{a} \xi_{(R)}^{b} d^{3} V+\int_{\Delta H} N_{R}\left\{|\sigma|^{2}+2|\zeta|^{2}\right\} d^{3} V .
$$

This is the key equation we were seeking to obtain quantitative expression for the change in the horizon area in fully dynamic processes. It will have several important applications. In the remainder of this sub-section we will focus on the first of these: its implications for the topology of $S$.

Let us first recall that the volume element $d^{3} V$ on $H$ can be written as $d^{3} V=N_{R}^{-1} d R d^{2} V$ where $d^{2} V$ is the area element on $S$. Therefore, the integral on the left hand side becomes:

$$
\int_{\Delta H} N_{R} \widetilde{\mathcal{R}} d^{3} V=\int_{R_{1}}^{R_{2}} d R \oint \widetilde{\mathcal{R}} d^{2} V=\mathcal{I}\left(R_{2}-R_{1}\right) .
$$

Here $R_{1}$ and $R_{2}$ are the (geometrical) radii of $S_{1}$ and $S_{2}$; we have used the Gauss-Bonnet theorem in the second step; and, $\mathcal{I}$ is the Gauss invariant of the closed, orientable 2-manifold $S$. (Our choice of lapse was made to enable this step in the calculation.) Substituting back in eq. (3.21) we obtain:

$$
\mathcal{I}\left(R_{2}-R_{1}\right)=16 \pi G \int_{\Delta H}\left(T_{a b}-\frac{\Lambda}{8 \pi G} g_{a b}\right) \widehat{\tau}^{a} \xi_{(R)}^{b} d^{3} V+\int_{\Delta H} N_{R}\left\{|\sigma|^{2}+2|\zeta|^{2}\right\} d^{3} V
$$

where we have used the definition (2.6) of $\bar{T}_{a b}$. The discussion of topology of $S$ is naturally divided in to three cases, depending on (the sign of) the cosmological constant.

Case 1: $\Lambda>0$. Now, since the stress energy tensor $T_{a b}$ is assumed to satisfy the dominant energy condition, the right side is manifestly positive definite. Since we already know that area increases along $\widehat{r}^{a}$, we have $R_{2}-R_{1}>0$. Hence it follows that $\mathcal{I}$ must 
be positive, whence the closed, orientable 2-manifolds $S$ are necessarily topological 2-spheres and $\mathcal{I}=8 \pi$. Eq. (3.23) now becomes:

$$
\begin{aligned}
\frac{R_{2}-R_{1}}{2 G} & =\int_{\Delta H}\left(T_{a b}-\frac{\Lambda}{8 \pi G} g_{a b}\right) \widehat{\tau}^{a} \xi_{(R)}^{b} d^{3} V \\
& +\frac{1}{16 \pi G} \int_{\Delta H} N_{R}\left\{|\sigma|^{2}+2|\zeta|^{2}\right\} d^{3} V .
\end{aligned}
$$

Case 2 : $\Lambda=0$. Now the right side of eq. (3.23) is necessarily non-negative. Hence, the topology of $S$ is either that of a 2-sphere (if the right side is positive) or that of a 2-torus (if the right side vanishes). As mentioned in Section IIC, this constraint on topology was obtained by Hayward [15] using a $2+2$ framework.

The torus topology can occur if and only if $T_{a b} \ell^{b}, \sigma_{a b}$ and $\zeta^{a}$ all vanish everywhere on $H$. Going back to Eq. (3.15), we conclude that the scalar curvature $\widetilde{\mathcal{R}}$ of $S$ must also vanish on every cross-section. ${ }^{3}$ Also, using the fact that $H$ is space-like, it now follows from eq. (2.1) that in this case $\mathcal{L}_{n} \Theta_{(\ell)}=0$ everywhere on $H$. Thus, in this case the dynamical horizon cannot be a FOTH. Furthermore, since $\Theta_{(\ell)}, \sigma_{a b}$ and $R_{a b} \ell^{b}$ all vanish on $H$, the Raychaudhuri equation now implies that $\mathcal{L}_{\ell} \Theta_{(\ell)}$ also vanishes. These strong restrictions imply that this is a degenerate case. For such horizons, although we know that the area must increase, eq. (3.23) trivializes whence we do not have a quantitative formula for the amount by which the area increases.

For generic dynamical horizons, the topology is $S^{2}$ and the quantitative relation is given by:

$$
\frac{1}{2 G}\left(R_{2}-R_{1}\right)=\int_{\Delta H} T_{a b} \widehat{\tau}^{a} \xi_{(R)}^{b} d^{3} V+\frac{1}{16 \pi G} \int_{\Delta H} N_{R}\left\{|\sigma|^{2}+2|\zeta|^{2}\right\} d^{3} V .
$$

Case 3: $\Lambda<0$. In this case there is no control on the sign of the right hand side of eq. (3.23). Hence, a priori any topology is permissible. Stationary solutions with quite general topologies are known for black holes which are locally asymptotically anti-de Sitter. Event horizons of these solutions are the potential asymptotic states of these dynamical horizons in the distant future.

In the remainder of this paper we will restrict our detailed calculations to the case of 2-sphere topology.

Remark: The above considerations provide an interesting constraint on the topology of marginally trapped surfaces if $\Lambda \geq 0$. As it stands, the discussion is restricted to the topology of cross-sections of dynamical horizons $H$. However, it is straightforward to generalize these results. Consider any 3 -manifold $\bar{H}$, foliated by compact 2 -surfaces $\bar{S}$. Then, by integrating (3.18) only on one leaf $\bar{S}$ of the foliation (rather than on $\Delta \bar{H}$ ), in place of (3.23) we obtain:

$$
\begin{aligned}
\mathcal{I} & =16 \pi G \int_{\bar{S}}\left(T_{a b}-\frac{\Lambda}{8 \pi G} g_{a b}\right) \widehat{\tau}^{a} \ell^{b} d^{2} V \\
& +\int_{\bar{S}}\left(|\sigma|^{2}+2|\zeta|^{2}-\frac{1}{2} \Theta_{(\ell)}\left(4 K-3 \Theta_{(\ell)}\right)+2 \widehat{r}^{a} D_{a} \Theta_{(\ell)}\right) d^{2} V .
\end{aligned}
$$

\footnotetext{
3 We thank J. Lewandowski for this observation. In view of these highly restrictive conditions, toroidal dynamical horizons appear to be unrelated to the toroidal topology of cross-sections of the event horizon discussed by Shapiro, Teukolsky, Winicour and others [19, 20].
} 
Now, if one leaf $\bar{S}_{o}$ of the foliation is marginally trapped and if $\widehat{r}^{a} D_{a} \Theta_{(\ell)} \geq 0$ on $\bar{S}_{o}$, we conclude that the topology of $\bar{S}_{o}$ must be that of a $S^{2}$ if $\Lambda>0$ and of a $S^{2}$ or a $T^{2}$ if $\Lambda=0 . T^{2}$ is a degenerate case in the sense explained above. Note that no assumption on the expansion $\Theta_{(n)}$ of $n^{a}$ has been used here.

Since $\bar{H}$ was arbitrary, we can also reach a conclusion on the topology of any marginally trapped surface $S$ in a space-time satisfying the dominant energy condition: either $S$ is topologically $S^{2}$ or $T^{2}$ or its (first order) deformation along any space-like, outward direction leads to a trapped surface. (A space-like direction $V^{a}$ will be said to be outward if $V^{a} \ell_{a}>0$.) In particular, then, if the topology is more complicated, the surface cannot lie on a trapping boundary. This is essentially Hawking's result [8].

\section{B. Gravitational energy flux}

Let us now interpret the various terms appearing in the area balance law. For simplicity of presentation, we will first focus on the case $\Lambda=0$ and comment on the $\Lambda \neq 0$ cases at the end.

The left side of eq. (3.25) provides us with the change in the horizon radius caused by the dynamical process under consideration. Since the expansion $\Theta_{(\ell)}$ vanishes, this is also the change in the Hawking mass as one moves from the cross section $S_{1}$ to $S_{2}$. The first integral on the right side of this equation is the flux $\mathcal{F}_{\text {matter }}^{(R)}$ of matter energy associated with the vector field $\xi_{(R)}^{a}$. The second term is purely geometrical and since it accompanies the term representing the matter energy flux, we propose to interpret it as the flux $\mathcal{F}_{\text {grav }}^{(R)}$ of $\xi_{(R)}^{a}$-energy in the gravitational radiation:

$$
\mathcal{F}_{\text {grav }}^{(R)}:=\frac{1}{16 \pi G} \int_{\Delta H} N_{R}\left\{|\sigma|^{2}+2|\zeta|^{2}\right\} d^{3} V .
$$

While the interpretation is naturally suggested by the area balance law (3.25), the key question is: Is this proposal physically viable? The purpose this sub-section is to argue that the answer is in the affirmative in the sense that it passes the 'standard' tests one uses to demonstrate the viability of the Bondi flux formula at null infinity.

- Gauge invariance: Since we did not have to introduce any structure, such as coordinates or tetrads, which is auxiliary to the problem, the expression is obviously gauge invariant. This is to be contrasted with definitions involving pseudo-tensors or background fields.

- Positivity: The energy flux is manifestly non-negative. In the case of the Bondi flux, positivity played a key role in the early development of the gravitational radiation theory. It was perhaps the most convincing evidence that gravitational waves are not coordinate artifacts but carry physical energy; as Bondi put it, 'one can heat water with them'.

It is surprising that a simple, manifestly non-negative expression can exist in the strong field regime of dynamical horizons. We did argue in section I that, since the energy is lost from the asymptotic region, one does expect an appropriately defined notion of gravitational energy flux across the surface of the black hole to be well-defined and positive. But the way in which the details work out is quite subtle. For example, since the issue is that of controlling signs, one may be tempted to conjecture that this positivity is a property of the black hole region where the expansion $\Theta_{(\ell)}$ of the outgoing normal is non-positive, i.e., of a definite sign. However, this conjecture turns out to be false! To show this, let us carry out the 
analysis of section III A on a general, foliated space-like surface $\bar{H}$. We can still obtain eq. (3.18) but, as is clear from (3.26), in place of the $\mathcal{F}_{\text {grav }}^{(R)}$ of $(3.27)$ the final expression would be:

$$
\overline{\mathcal{F}}_{\text {grav }}^{(R)}:=\frac{1}{16 \pi G} \int_{\Delta H} N_{R}\left\{|\sigma|^{2}+2|\zeta|^{2}+\frac{1}{2} \Theta_{(\ell)}\left(4 K-3 \Theta_{(\ell)}\right)+2 \widehat{r}^{a} D_{a} \Theta_{(\ell)}\right\} d^{3} V .
$$

The key point is that if $\bar{H}$ is not a dynamical horizon, the sign of the last two terms cannot be controlled, not even when $\bar{H}$ lies in the black hole region and is foliated by trapped (rather than marginally trapped) surfaces $\bar{S}$. Thus, the positivity of $\mathcal{F}_{\text {grav }}^{(R)}$ is a rather subtle property, not shared by 3-surfaces which are foliated by non-trapped surfaces, nor those which are foliated by trapped surfaces; one needs a foliation precisely by marginally trapped surfaces. Thus, the property is delicately matched to the definition of dynamical horizons. This is but one instance of the mysterious ability of Einstein's equations to realize physical expectations through geometrical structures in completely unforeseen and subtle ways. ${ }^{4}$

- Locality: All fields used in it are defined by the local geometrical structures on crosssections of $H$. This is a non-trivial property, shared also by the Bondi-flux formula. However, it is not shared in other contexts. For example, the proof of the positive energy theorem by Witten [21] provides a positive definite energy density on Cauchy surfaces. But since it is obtained by solving an elliptic equation with appropriate boundary conditions at infinity, this energy density is a highly non-local function of geometry. Locality of $\mathcal{F}_{\text {grav }}^{(R)}$ enables to associate it with the energy of gravitational waves instantaneously falling across any cross section $S$.

- Vanishing in spherical symmetry: The fourth criterion is that the flux should vanish in presence of spherical symmetry. Suppose the cross-sections $S$ of $H$ are spherically symmetric. Since the only spherically symmetric vector field and trace-free, second rank tensor field on a 2-sphere are the zero fields, $\sigma_{a b}=0$ and $\zeta^{a}=0$.

- Relation to perturbation theory: The fifth criterion comes from perturbation theory. One can envisage a situation in which the dynamical horizon is, in an appropriate physical sense, weakly dynamical. In this case, it can be regarded as a perturbation of a nonexpanding horizon [6] (see section VI). It is then natural to ask if in this case the gravitational flux (3.27) reduces to the expression derived from perturbation theory off Kerr horizons. The answer is in the affirmative.

- Balance law: The Bondi-Sachs energy flux also has the important property that there is a locally defined notion of the Bondi-energy $E(C)$ associated with any 2-sphere crosssection $C$ of future null infinity and the difference $E\left(C_{1}\right)-E\left(C_{2}\right)$ equals the Bondi-Sachs flux through the portion of null infinity bounded by $C_{2}$ and $C_{1}$. Does the expression (3.27) share this property? The answer is in the affirmative: as noted in the beginning of this subsection, the integrated flux is precisely the difference between the locally defined Hawking mass associated with the cross-section. In Section V we will extend these considerations to include angular momentum.

\footnotetext{
${ }^{4}$ Some of the well-known examples are: the well-posedness of the Cauchy problem; the positive energy theorems at spatial and null infinity; positivity of the Bondi flux at null infinity; and more open-ended issues such as cosmic censorship and Penrose inequalities. Not only did the list of considerations that led Einstein to his field equations not include these issues but even the physical relevance of most of them was not appreciated for decades after the discovery of general relativity. Yet, quite mysteriously, the field equations incorporate them correctly!
} 
- Hamiltonian interpretation: Finally, the Bondi-Sachs energy flux has an additional attractive property which supports its interpretation, although it is not a direct, physical, viability criterion: Using a Hamiltonian framework, one can show that it is the generator of a Bondi-Metzner-Sachs time-translation on the gravitational phase space [11, 12]. Does the gravitational flux (3.27) also enjoy this property? Recently, Booth and Fairhurst [22] have shown that the answer is in the affirmative.

It is very surprising that there should be a meaningful expression for the gravitational energy flux in the strong field regime where gravitational waves can no longer be envisaged as ripples on a flat space-time. Taken together, the properties discussed above provide a strong support in favor of the interpretation of (3.27) as the $\xi_{(R)}$-energy flux of carried by gravitational waves into the portion $\Delta H$ of the dynamical horizon. Nonetheless, it is important to continue to think of new criteria and make sure that (3.27) passes these tests. For instance, in physically reasonable, stationary, vacuum solutions to Einstein's equations, one would expect that the flux should vanish. However, on dynamical horizons the area must increase. Thus, one is led to conjecture that these space-times do not admit dynamical horizons. While special cases of this conjecture have been proved, a general proof is still lacking.

So far, we have set the cosmological constant $\Lambda$ to zero. Even when $\Lambda$ is non-zero, it seems natural to continue to interpret (3.27) as the $\xi_{(R)}$-energy flux of carried by gravitational waves into the portion $\Delta H$ of the dynamical horizon. However, now there is an additional, purely geometrical contribution to the area change of eq. (3.24) coming from the cosmological repulsion or attraction induced by the cosmological constant. If $\Lambda$ is positive, the area of the cross-sections $S$ of $H$ would continue to grow just because of the cosmological expansion even when there is no flux of gravitational or mater energy across $\Delta H$, while if $\Lambda$ is negative, it would decrease.

To conclude this sub-section, we will comment on some issues related to the physical interpretation of the flux formula. Note first that the flux refers to a specific vector field $\xi_{(R)}^{a}$ and measures the change in the Hawking mass associated with the cross-sections. This need not be a good measure of the physical mass in presence of angular momentum (see section $\mathrm{V}$ ). Secondly, one can envisage a situation in which the portion $\Delta H$ bounded by $S_{2}$ and $S_{1}$ of a dynamical horizon admits two distinct foliations in the both of which share the leaves $S_{1}$ and $S_{2}$, or, a situation in which two distinct dynamical horizons $H_{1}$ and $H_{2}$ share the 2 -spheres $S_{2}$ and $S_{1}$. In these cases, the observer fields $\xi_{(R)}$ are distinct. Although the total fluxes corresponding to the two fields do agree - they are given by the change in horizon radius as one goes from $S_{1}$ to $S_{2}$ - the split between the matter contribution and the gravitational wave contribution would be different. This is not surprising because we are in a strong field region and it is not inappropriate for two observers to disagree on how much energy is contained in matter and how much in gravitational radiation. Indeed, a priori, what is surprising is that the sum of the two contributions is the same, i.e., there is an area balance law. Nonetheless, while interpreting fluxes, the fact that the energy refers to specific observers defined on $H$ is an important caveat that should be kept in mind.

Next, let us consider the various terms in the integrand of our flux formula (3.27). The presence of the shear term $|\sigma|^{2}$ seems natural from one's expectations based on perturbation theory at the event horizon of the Kerr family [23, 24]. What about the term $|\zeta|^{2}$ ? Since $\zeta^{a}=\widetilde{q}^{a n} \widehat{r}^{m} \nabla_{m} \ell_{n}$, this term could arise only because $H$ is space-like rather than null: On a null surface, the analog of $\widehat{r}^{a}$ is parallel to $\ell^{a}$, whence the analog of $\zeta^{a}$ vanishes identically. To bring out this point, let us consider a more general case than the one considered in this 
paper and allow the cross-sections $S$ to lie on a horizon which is partially null and partially space-like. Then, using a $2+2$ formulation used by Hayward, one can conclude that flux on the null portion is given entirely by the term $|\sigma|^{2}[18]$. However, on the space-like portion, the term $|\zeta|^{2}$ does not in general vanish. Indeed, on a dynamical horizon, it cannot vanish in presence of rotation: the angular momentum is given by the integral of $\zeta^{a} \varphi_{a}$, where $\varphi^{a}$ is the rotational symmetry.

\section{Generalization of the area balance law}

At future null infinity $\mathbb{I}^{+}$, there is a well-defined, 4-dimensional translation sub-group $\mathbb{T}$ of the asymptotic symmetry group (called the Bondi-Metzner-Sachs group) and there is a well-defined notion of energy associated with each time translation in $\mathbb{T}$. Observers following these vector fields can be physically interpreted as the asymptotically inertial ones. In sections III A and III B, we associated energy with observers following the vector fields $N_{R} \ell^{a}$. Are there more general families with which we can similarly assign a notion of energy?

At the dynamical horizon $H$ we are in the strong field regime, whence there is no longer a universal group of horizon symmetries. But we can build intuition from the well-developed theory of weakly-isolated horizons $\Delta$. In this case, to begin with, one encounters three universality classes of horizon symmetries [7]. Physically, the most interesting case is that of type II isolated horizons in which the symmetry group is 2-dimensional, with generators $c \ell^{a}+$ $\Omega \varphi^{a}$, where $c, \Omega$ are constants, while $\ell^{a}, \varphi^{a}$ are tangential to $\Delta$ and generate a combination of a time translation and a rotation. In globally stationary, axi-symmetric space-times, these are restrictions to $\Delta$ of the two Killing fields but generically they are defined just at the horizon. Nonetheless, they can be used very effectively in the Hamiltonian framework to introduce the notion of the horizon energy and angular momentum. For dynamical horizons $H$, it is natural to extend these notions in such a way that when $H$ reaches equilibrium and becomes an isolated horizon, the dynamical horizon framework tends to the isolated horizon one. An obvious strategy is to make the coefficients $c$ and $\Omega$ dynamical, i.e., $R$-dependent. In this sub-section we will focus only on the analog of the coefficient $c$, i.e., ignore rotation as in [6]. Inclusion of rotation and the analog of $\Omega$ will be carried out in section $\mathrm{V}$.

Let us then generalize our vector fields $N_{R} \ell^{a}$ as follows: use, in place of $R$, a general function $r(R)$. Recall first that $N_{R}$ satisfies $D_{a} R=N_{R} \widehat{r}_{a}$ so that we have $N_{R} d^{3} V=d R d^{2} V$. Therefore, for more general functions $r(R)$ which are constant on each leaf $S$ of the foliation, we are led to choose $N_{r}$ through $D_{a} r=N_{r} \widehat{r}_{a}$. If we use a different radial function $r^{\prime}$, then the lapse is rescaled according to the relation

$$
N_{r^{\prime}}=\frac{d r^{\prime}}{d r} N_{r}
$$

Thus, although the lapse itself will in general be a function of all three coordinates on $H$, the relative factor between any two permissible lapses can be a function only of $r$. This is the simplest generalization that seems appropriate to the transition from isolated to dynamical horizons.

Given a lapse $N_{r}$, following the terminology used in the isolated horizon framework, the resulting vector fields by $\xi_{(r)}^{a}:=N_{r} \ell^{a}$ will be said to be permissible. Thus, $\xi_{(R)}^{a}$ used in section III A is just one permissible vector field which (on dimensional grounds) happens to be the convenient one to relate the change $R_{2}-R_{1}$ in the horizon radius to the flux of 
energy across $\Delta H$. By repeating the calculation of section III A, it is easy to arrive at a generalization of (3.24) for any permissible vector field:

$$
\left(\frac{r_{2}}{2 G}-\frac{r_{1}}{2 G}\right)=\int_{\Delta H} \bar{T}_{a b} \widehat{\tau}^{a} \xi_{(r)}^{b} d^{3} V+\frac{1}{16 \pi G} \int_{\Delta H} N_{r}\left\{|\sigma|^{2}+2|\zeta|^{2}\right\} d^{3} V,
$$

where the constants $r_{1}$ and $r_{2}$ are values the function $r$ assumes on the fixed cross-sections $S_{1}$ and $S_{2}$. (Note, incidentally, that the lapse $N_{r}$ may well vanish on open regions. It may also be negative in which case we would have $r_{2}<r_{1}$.) This generalization of (3.23) will be useful in section VI.

Here, we simply note a special case of physical interest: $r=4 \pi R^{2}$. In this case, (3.30) directly gives us a formula for the change in the horizon area (rather than in the horizon radius):

$$
\left(\frac{a_{2}}{4 G}-\frac{a_{1}}{4 G}\right)=\frac{1}{2} \int_{\Delta H} \bar{T}_{a b} \widehat{\tau}^{a} \xi_{(r)}^{b} d^{3} V+\frac{1}{32 \pi G} \int_{\Delta H} N_{r}\left\{|\sigma|^{2}+2|\zeta|^{2}\right\} d^{3} V
$$

Note however that, as is expected from dimensional reasons, the right hand side does not have the interpretation of the energy flux across $\Delta H$ even in the case $\Lambda=0$. However, since black hole thermodynamics tells us that the (leading contribution to the) entropy is given by $a / 4 \ell_{\mathrm{Pl}}^{2}$, one may wish to interpret the right hand side as the entropy flux through $\Delta H$ (in the $\hbar=1$ units).

Remark: In the definition of a dynamical horizon, we required $\Theta_{(n)}<0$ which guaranteed that $|D R| \neq 0$, i.e., that $R$ is a good coordinate on $H$. This was used in the derivation of (3.23) in section III A. However, we can weaken the definition and ask only that $\Theta_{(n)} \leq 0$. In this case, we can introduce a function $x$ such that the marginally trapped 2-surfaces are labelled by $x=$ const and $|D x| \neq 0$ and repeat the calculations of section III A to obtain the analog of (3.23) in which $R$ is replaced by $x$. We can then note that although $R$ need not be a good coordinate on $H$, it is nonetheless a smooth function of the coordinate $x$ whence, the calculation of this sub-section can be repeated to obtain the area balance (3.23). Thus, the area balance law holds also under the weaker assumption $\Theta_{(n)} \leq 0$. If $\Theta_{(n)}>0$, we can reverse the argument to get an area decrease law appropriate for white holes.

\section{ANGULAR MOMENTUM}

To obtain the integral version of the first law (1.1), we need the notion of angular momentum and angular momentum flux. It turns out that the angular momentum analysis is rather straight forward and is, in fact, applicable to an arbitrary space-like hypersurface. Fix any vector field $\varphi^{a}$ on $H$ which is tangential to all the cross-sections $S$ of $H$. Contract both sides of $(2.5)$ with $\varphi^{a}$. Integrate the resulting equation over the region $\Delta H$, perform an integration by parts and use the identity $\mathcal{L}_{\varphi} q_{a b}=2 D_{(a} \varphi_{b)}$ to obtain

$$
\frac{1}{8 \pi G} \oint_{S_{2}} K_{a b} \varphi^{a} \widehat{r}^{b} d^{2} V-\frac{1}{8 \pi G} \oint_{S_{1}} K_{a b} \varphi^{a} \widehat{r}^{b} d^{2} V=\int_{\Delta H}\left(T_{a b} \widehat{\tau}^{a} \varphi^{b}+\frac{1}{16 \pi G} P^{a b} \mathcal{L}_{\varphi} q_{a b}\right) d^{3} V
$$

where, as before, $P^{a b}:=K^{a b}-K q^{a b}$. (Note that we could replace $\bar{T}_{a b}$ with $T_{a b}$ because $g_{a b} \widehat{\tau}^{a} \varphi^{b}=0$. Thus the cosmological constant plays no role in this section.) It is natural 
to identify the surface integrals with the generalized angular momentum $J^{\varphi}$ associated with cross-sections $S$ and set

$$
J_{S}^{\varphi}=-\frac{1}{8 \pi G} \oint_{S} K_{a b} \varphi^{a} \widehat{r}^{b} d^{2} V
$$

where we have chosen the overall sign to ensure compatibility with conventions normally used in the asymptotically flat context. The term 'generalized' emphasizes the fact that the vector field $\varphi^{a}$ need not be an axial Killing field even on $S$; it only has to be tangential to our cross-sections.

The flux of this angular momentum due to matter fields and gravitational waves are respectively

$$
\begin{aligned}
\mathcal{J}_{\text {matter }}^{\varphi} & =-\int_{\Delta H} T_{a b} \widehat{\tau}^{a} \varphi^{b} d^{3} V \\
\mathcal{J}_{\text {grav }}^{\varphi} & =-\frac{1}{16 \pi G} \int_{\Delta H} P^{a b} \mathcal{L}_{\varphi} q_{a b} d^{3} V
\end{aligned}
$$

and we get the balance equation

$$
J_{S_{2}}^{\varphi}-J_{S_{1}}^{\varphi}=\mathcal{J}_{\text {matter }}^{\varphi}+\mathcal{J}_{\text {grav }}^{\varphi} \cdot
$$

As expected, if $\varphi^{a}$ is a Killing vector of the three-metric $q_{a b}$, then the gravitational angular momentum flux vanishes: $\mathcal{J}_{\mathrm{g}}^{\varphi}=0$. For the discussion of the integral version of the first law, it is convenient to introduce the angular momentum current

$$
j^{\varphi}:=-K_{a b} \varphi^{a} \widehat{r}^{b}
$$

so that the angular momentum formula becomes

$$
J_{S}^{\varphi}=\frac{1}{8 \pi G} \oint_{S} j^{\varphi} d^{2} V
$$

We conclude with four remarks:

i. Interpretation of $\zeta^{a}$ : We can use the expression of $J_{S}^{\varphi}$ to interpret the vector field $\zeta^{a}$ which features in the gravitational energy flux: $\zeta^{a}=0$ on $H$ if and only if $J_{S}^{\varphi}=0$ for every $\varphi^{a}$ which is divergence-free (i.e. preserves the volume-element) on $S$.

ii. Relation to other expressions: Let us restrict ourselves to vector fields $\varphi^{a}$ which are divergence-free on each cross-section $S$. The angular momentum $J_{S}^{\varphi}$ associated with these $\varphi^{a}$ have the following interesting property. Fix a cross-section $S$ of $H$ and consider an asymptotically flat, partial Cauchy surface $M$ in the space-time $\mathcal{M}$ with inner boundary $S$. Denote its Cauchy data by $\left(\bar{q}_{a b}, \bar{K}_{a b}\right)$. Then, we can extend $\varphi^{a}$ to a vector field $\phi^{a}$ which is an asymptotic rotational symmetry of $\left(M, \bar{q}_{a b}\right)$ and repeat the above calculation by replacing $\Delta H$ with $M$. The surface integral at infinity is then the standard ADM angular momentum associated with $\phi^{a}$. The angular momentum assigned to $S$ is:

$$
\bar{J}_{S}^{\varphi}=-\frac{1}{8 \pi G} \oint_{S} \bar{K}_{a b} \varphi^{a} \bar{r}^{b} d^{2} V
$$

where $\bar{r}^{a}$ is the unit normal to $S$ in $M$. By expressing $K_{a b}$ and $\bar{K}_{a b}$ in terms of $\nabla_{a} \ell_{b}$ and $\nabla_{a} n_{b}$, it is straightforward to show that $J_{S}^{\varphi}=\bar{J}_{S}^{\varphi}$. Thus, given a divergence-free $\varphi^{a}$ on $S$, the notion of angular momentum associated with $S$ is unambiguous. Finally, if $\varphi^{a}$ is the restriction to $S$ of a space-time Killing vector defined in a neighborhood of $S$, one can define the angular momentum via Komar integral and it agrees with $J_{S}^{\varphi}$. 
iii. Dependence on $\varphi^{a}$ : In the above calculation we did not assume that $\varphi^{a}$ is a Killing field on $H$. However, $J_{S}^{\varphi}$ would represent the physical angular momentum at the 'instant' $S$ only if $\varphi^{a}$ is a Killing field of at least $\left(S, \widetilde{q}_{a b}\right)$. Suppose $\varphi^{a}$ has this property both on $S_{1}$ and $S_{2}$, but not on all of $\Delta H$. Still, because of the balance law (4.1), the total flux is well-defined and is in fact independent of the way in which $\varphi^{a}$ is extended off $S_{1}$ and $S_{2}$.

iv. Gauge fields: We indicated in section IIC that there are subtleties associated with gauge fields. Considerations of angular momentum illustrate this point. In the above treatment, we just interpreted $\int_{\Delta H} T_{a b} \widehat{\tau}^{a} \varphi^{b} d^{3} V$ as the flux of matter angular momentum across $\Delta H$. But a priori there is some freedom to shuffle terms between the 3 -dimensional flux integrals and the 2-dimensional 'angular momentum charge' integrals. Our choice ensures that, as at infinity, the 2-sphere 'angular momentum charge' integrals $J_{S}^{\varphi}$ depend only on geometric fields and not on matter. However Hamiltonian considerations often show that, in order for angular momentum to be the generator of rotations on the phase space, such a reshuffling is in fact necessary in the case of gauge fields. Thus, the 'angular momentum charge' integral can in fact depend on gauge fields as well. (In the case of isolated horizons, this is demonstrated in detail in [7].) The required shuffling will not affect any of the equations but would change interpretations of terms in presence of gauge matter fields.

\section{INTEGRAL VERSION OF THE FIRST LAW AND THE HORIZON MASS}

This section is divided in to three parts. In the first we obtain an integral generalization of the first law (1.1). In the second, we restrict ourselves to axi-symmetric dynamical horizons and introduce, for each cross-section $S$, a canonical notion of energy (which may be interpreted as the instantaneous mass) and derive a balance law. In the third, we discuss the distinction between laws of 'black hole mechanics' and of 'black hole thermodynamics'.

\section{A. Generalization of the first law of black hole mechanics}

Let us now combine the results of sections III and IV to obtain the physical process version of the first law on $H$. As in section III B, we will first consider the case $\Lambda=0$ and then comment on the role played by the non-zero cosmological constant.

To begin with, let us ignore angular momentum and consider the vector field $\xi_{(R)}^{a}$ of section III A. For each cross-section $S$ of $H$, there is a well-defined notion of horizon energy $E^{\xi_{(R)}}(S)$ (given just by the Hawking mass). Because of the influx of matter and gravitational energy, $E^{\xi_{(R)}}$ will change by an amount $\Delta E^{\xi_{(R)}}=\mathcal{F}_{\text {matter }}^{(R)}+\mathcal{F}_{\text {grav }}^{(R)}$ as we move from a cross-section $S_{1}$ to another cross-section $S_{2}$. Then, the infinitesimal form of (3.25),

$$
\frac{d R}{2 G}=d E^{\xi_{(R)}},
$$

suggests that we define effective surface gravity $\bar{\kappa}_{R}$ associated with $\xi_{(R)}^{a}$ as

$$
\bar{\kappa}_{R}:=\frac{1}{2 R}
$$


so that the infinitesimal expression is recast into the familiar form

$$
\left(\frac{\bar{\kappa}_{R}}{8 \pi G}\right) d a=d E^{\xi_{(R)}}
$$

where $a$ is the area of a generic cross-section. (This conclusion could also have been reached from (3.31)). For a general choice of the radial function $r$, the infinitesimal version of (3.30) yields a generalized first $l a w^{5}$ :

$$
\frac{\bar{\kappa}_{r}}{8 \pi G} d a=d E^{\xi_{(r)}}
$$

provided we define the effective surface gravity $\bar{\kappa}_{r}$ of $\xi_{(r)}^{a}$ by

$$
\bar{\kappa}_{r}=\frac{d r}{d R} \bar{\kappa}_{R} \quad \text { where } \quad \xi_{(r)}^{a}=N_{r} \ell^{a}=\frac{d r}{d R} \xi_{(R)}^{a} .
$$

Note that this rescaling freedom in surface gravity is completely analogous to the rescaling freedom which exists for Killing horizons, or, more generally, isolated horizons [6, 7]. There, on the horizon $\ell^{a}$ can be rescaled by a constant and surface gravity rescales by the same constant. The new feature in the present case is that we have the freedom to rescale $N_{R} \ell^{a}$ and the surface gravity by a function of the radius $R$ rather than just a constant. This is just what one would expect in a dynamical situation since $R$ plays the role of time along $H$. Finally, note that the differentials appearing in (5.4) are the actual variations of physical quantities along the dynamical horizon due to an infinitesimal change in $r$. This is to be contrasted with derivations of the first law based on phase space variations [6, 7, 25], where one compares quantities defined on distinct (isolated or Killing) horizons belonging to distinct space-times. Since quantities defined in distinct equilibrium configurations are compared, there one obtains a passive form of the first law. By contrast, (5.4) is an active or a physical process version of the first law. Hence (3.30) is a finite version of the first law in absence of rotation. As in the case of isolated horizons [6], even in absence of rotation, there are many permissible vector fields and each gives rise to a first law.

Next, let us include rotation. As discussed in section III C, the general strategy is motivated by the isolated horizon framework. Pick a vector field $\varphi^{a}$ on $H$ such that $\varphi^{a}$ is tangent to the cross-sections of $H$, has closed orbits and has affine length $2 \pi{ }^{6}$ (At this point, $\varphi^{a}$ need not be a Killing vector of $q_{a b}$.) The isolated horizons considerations suggest that it is now appropriate to replace $\xi_{(r)}^{a}$ by vector fields $t^{a}$ which are of the form $t^{a}=N_{r} \ell^{a}-\Omega \varphi^{a}$ where $N_{r}$ is a permissible lapse associated with a radial function $r$ and $\Omega$ an arbitrary function of $R$. (On an isolated horizon, the analogs of these two fields are constants.) Such vector fields $t^{a}$ will be said to be permissible. Let us now evaluate the quantity $\int_{\Delta H} T_{a b} \widehat{\tau}^{a} t^{b} d^{3} V$ by taking a linear combination of (3.30) and (4.1). We obtain:

$$
\begin{aligned}
& \frac{r_{2}-r_{1}}{2 G}+\frac{1}{8 \pi G}\left\{\oint_{S_{2}} \Omega j^{\varphi} d^{2} V-\oint_{S_{1}} \Omega j^{\varphi} d^{2} V-\int_{\Omega_{1}}^{\Omega_{2}} d \Omega \oint_{S} j^{\varphi} d^{2} V\right\}= \\
& \int_{\Delta H} T_{a b} \widehat{\tau}^{a} t^{b} d^{3} V+\frac{1}{16 \pi G} \int_{\Delta H} N_{r}\left(|\sigma|^{2}+2|\zeta|^{2}\right) d^{3} V-\frac{1}{16 \pi G} \int_{\Delta H} \Omega P^{a b} \mathcal{L}_{\varphi} q_{a b} d^{3} V(5.6)
\end{aligned}
$$

\footnotetext{
${ }^{5} E^{\xi_{(r)}}$ has the dimension of energy only if $r$ has the same dimension as $R$. In the following discussion, we will assume this to be the case.

${ }^{6}$ More precisely, $\varphi^{a}$ is a globally defined Killing field for some metric - not necessarily the physical one, $\widetilde{q}_{a b}$ - on each 2-sphere cross-section $S$ of $H$.
} 
These are our balance equations in presence of angular momentum. There are infinitely many balance equations because there are infinitely many permissible vector fields. In section V B, we will show that, when the horizon metric $q_{a b}$ is axi-symmetric, one can choose a preferred vector field $t_{o}^{a}$ (which is adapted to the Kerr time-translation Killing field in a precise sense.) For this vector field, given a cross-section $S$, we will provide an explicit expression of the energy $E_{S}^{t_{o}}$ such that the left side of Eq. (5.6) can be re-expressed as the difference $E_{S_{2}}^{t_{o}}-E_{S_{1}}^{t_{o}}$, whence we are led to a preferred balance equation:

$$
\begin{aligned}
E_{S_{2}}^{t_{o}}-E_{S_{1}}^{t_{o}}=\int_{\Delta H} T_{a b} \widehat{\tau}^{a} t_{o}^{b} d^{3} V & +\frac{1}{16 \pi G} \int_{\Delta H} N_{o}\left(|\sigma|^{2}+2|\zeta|^{2}\right) d^{3} V \\
& -\frac{1}{16 \pi G} \int_{\Delta H} \Omega_{o} P^{a b} \mathcal{L}_{\varphi} q_{a b} d^{3} V
\end{aligned}
$$

Let us return to the general case considered in Eq. (5.6). Assuming there is a welldefined notion $E^{t}$ of the horizon energy at each cross-section, with the right side of (5.6) its flux, we can now obtain the first law for mechanics for dynamical horizons. Let us restrict ourselves to infinitesimal $\Delta H$. Then, the three terms in the curly brackets combine to give $d(\Omega J)-J d \Omega$ and eq. (5.6) reduces to

$$
\frac{d r}{2 G}+\Omega d J \equiv \frac{\bar{\kappa}_{r}}{8 \pi G} d a+\Omega d J=d E^{t} .
$$

This is just the familiar first law but now in the setting of dynamical horizons. Since the differentials in this equation are variations of physical quantities along $H$, this can be viewed as a physical process version of the first law of black hole mechanics. Note that for each allowed choice of lapse $N_{r}$, angular velocity $\Omega(r)$ and vector field $\varphi^{a}$ on $H$, we obtain a permissible time vector field $t^{a}=N_{r} \ell^{a}-\Omega \varphi^{a}$ and a corresponding first law. For isolated horizons $[6,7]$ the situation is similar; there are infinitely many permissible vector fields and a first law for each of them. The main difference is that we are now in a dynamical situation and (5.8) tells us what happens instantaneously on the dynamical horizon (at the 'instant' represented by the cross-section $S$ ). The first law in $[6,7]$ describes transitions from one equilibrium situation to a nearby one and refers to the isolated horizon as a whole. Again, the generalization from that time independent situation consists of allowing the lapse and the angular velocity to become $R$-dependent, i.e., dynamical. Therefore, for vector fields $t^{a}$ for which there is a satisfactory notion of horizon energy $E^{t}$ (as for $t^{a}=t_{o}^{a}$ introduced in section VB), eq. (5.6) yields an integral, physical process version generalization of the familiar, differential first laws of isolated horizon mechanics:

$$
\frac{r_{2}-r_{1}}{2 G}+\frac{1}{8 \pi G}\left\{\oint_{S_{2}} \Omega j^{\varphi} d^{2} V-\oint_{S_{1}} \Omega j^{\varphi} d^{2} V-\int_{\Omega_{1}}^{\Omega_{2}} d \Omega \oint_{S} j^{\varphi} d^{2} V\right\}=E_{S_{2}}^{t}-E_{S_{1}}^{t}
$$

Thus, if a suitable notion of horizon energy $E_{S}^{t}$ can be found, the same equation (5.6) can be used to obtain an energy balance equation (5.7) similar to that of Bondi and Sachs, but now at the dynamical horizon, and an integral generalization (5.9) of the active form of the first law of black hole mechanics.

Finally, let us consider the case when the cosmological constant is non-zero. Then, the integral version of the first law is given simply by replacing $T_{a b}$ in eq. (5.6) by $\bar{T}_{a b}$. In the infinitesimal version, we now obtain

$$
\frac{\bar{\kappa}_{r}}{8 \pi G}\left[1-\Lambda R^{2}\right] d a+\Omega d J=d E^{t}
$$


Thus, the only effect that a cosmological constant has is to modify the expression of effective surface gravity. This is completely analogous to what happens to the standard first law on Killing or isolated horizons.

We conclude with two remarks:

i. More general permissible vector fields: Since we selected the vector fields $t^{a}$ using intuition derived from isolated horizons, we were led to ask that $N_{r} / N_{R}$ and $\Omega$ be functions only of $R$. But it is rather easy to allow more general $N, \Omega$ and thus extend the notion of permissible vector fields. Set $\bar{t}^{a}=N \ell^{a}-\Omega \varphi^{a}$, where $N$ is any smooth function on $H$, not necessarily tied to a radial function $r$. Then, we obtain an obvious generalization of the balance equation (5.6). Furthermore, we can set the effective surface gravity to be

$$
\bar{\kappa}_{\bar{\xi}}=\left(\frac{1}{8 \pi} \oint N N_{R}^{-1} \widetilde{\mathcal{R}} d^{2} V\right) \bar{\kappa}_{R}
$$

and again obtain the first law (5.8) with $\kappa_{r}$ replaced by $\kappa_{\bar{\xi}}$.

Our restriction on $\Omega$ being only a function of $R$ corresponds to considering rigidly rotating fields $t^{a}$ (where, however, the angular speed of rotation is allowed to vary as one moves from one cross-section to another). This restriction is necessary to recover the familiar infinitesimal form of the first law and also for the definition of the horizon energy in section VB. However, as far as the integral first law is concerned, one can easily accommodate differential rotation by allowing $\Omega$ to be a function also of angular coordinates.

ii. The $\Theta_{(n)} \leq 0$ case: It is easy to verify that the main result of this section goes through even if the condition $\Theta_{(n)}<0$ is weakened to allow $\Theta_{(n)} \leq 0$. The reasoning is the same as that in the remark at the end of section III.

\section{B. Horizon mass}

Recall first the situation at null infinity. Given a time translation $t$ in the Bondi-MetznerSachs group and a cross-section $\mathbb{S}$ of $\mathbb{I}^{+}$, we can define Bondi-energy $E_{S}^{t}$ such that the difference between the energy associated with any two cross-sections equals the Bondi-flux through the region of $\mathbb{I}^{+}$they bound [10-12]. On dynamical horizons, the right side of eq. (5.6) provides us with the analog of the Bondi-flux. It is natural to ask if there is also a satisfactory notion of energy $E_{S}^{t}$ associated with each cross-section $S$. In this sub-section, we will address this issue using dual considerations: finding preferred fields for which a mathematically viable notion of $E_{S}^{t}$ exists and admits a satisfactory physical interpretation. We will first restrict ourselves to the case $\Lambda=0$ and show that the both goals can be met for axi-symmetric dynamical horizons.

Given any permissible vector field $t^{a}$ on $H$, we can just solve the ordinary differential equation on $H$,

$$
\frac{d E^{t}}{d R}=\frac{R}{G} \bar{\kappa}_{r}(R)+\Omega \frac{d J}{d R},
$$

derived from (5.8), and obtain an expression $E_{S}^{t}$ on any cross section $S$. But in general the result will not be expressible in terms of geometric quantities defined locally on $S$. If it is, we will have a mathematically viable notion of $E_{S}^{t}$. Our second requirement is that the resulting $E_{S}^{t}$ should have a direct physical interpretation. 
The first example is provided by dynamical horizons $H$ on which the intrinsic metric $q_{a b}$ of $H$ is spherically symmetric. Then, it is natural to choose $\Omega=0$ and $R$ as the radial coordinate so that the preferred vector field is $t_{o}^{a}=N_{R} \ell^{a}$ with effective surface gravity $\bar{\kappa}_{R}=1 / 2 R$. In this case, the integration of the flux yields:

$$
E_{S}^{t}=\frac{R}{2 G}
$$

where the integration constant has been chosen such that $E_{S}^{t}$ tends to the isolated horizon mass when the matter flux vanishes and the horizon reaches equilibrium. Since we arrived at this expression by integrating the differential equation (5.8), and since the right side of this equation does not refer to matter fields at all, the expression of $E_{S}^{t}$ is purely geometric. In fact, since the expansion $\Theta_{(\ell)}$ vanishes on $S$, as noted before, $E_{S}^{t}$ is precisely the Hawking mass of $S$. In the spherically symmetric case, this is a physically viable measure of energy in the black hole; thus both our goals are met. Furthermore, by restricting the balance law (5.6) to this case, we conclude:

$$
E_{S_{2}}^{t}-E_{S_{1}}^{t}=\int_{\Delta H} T_{a b} \widehat{\tau}^{a} t^{b} d^{3} V .
$$

Thus, (5.6) has a clear-cut interpretation in this case: the flux of gravitational energy vanishes, and the increase in $E^{t}(S)$ is fully accounted for by the matter flux $\mathcal{F}_{\text {matter }}^{(R)}$. Note that this was obtained assuming spherical symmetry only of $\left(H, q_{a b}\right)$.

Beyond spherical symmetry, the gravitational energy flux would not be zero, whence the balance equation will be non-trivially generalized. We can begin with distorted but nonrotating dynamical horizons, i.e., ones on which the angular momentum current density $\widetilde{q}^{a b} K_{b c} \widehat{r}^{c}$ vanishes. Again, it is appropriate to set $\Omega=0$. Furthermore, from isolated horizon considerations, we know that the distortion does not affect surface gravity [6]. Therefore we can again set $r=R$. Thus, the discussion is reduced to that in the spherically symmetric case. Again, the isolated horizon framework supports the interpretation of the Hawking mass as the horizon mass in this case as well. The difference from spherical symmetry is that now there may be gravitational radiation. Thus, in the distorted case, the balance equation derived from (5.6) is more general:

$$
E_{S_{2}}^{t}-E_{S_{1}}^{t}=\int_{\Delta H} T_{a b} \widehat{\tau}^{a} t^{b} d^{3} V+\frac{1}{16 \pi G} \int_{\Delta H} N_{R}\left(|\sigma|^{2}+2|\zeta|^{2}\right) d^{3} V
$$

(However, because the angular momentum current vanishes, the expression of $\zeta^{a}$ simplifies to: $\zeta^{a}=\widetilde{q}^{a b} D_{b} \ln N_{R}$.)

Finally let us incorporate rotation. Physically, the most interesting case is the one in which $q_{a b}$ is axi-symmetric, with $\varphi^{a}$ as its axial Killing vector. (In what follows, we will work with this fixed $\varphi^{a}$. The dependence on $\varphi^{a}$ of various physical quantities such as the angular momentum will now be dropped.) To specify a preferred vector field $t_{o}^{a}$, we need to specify $\bar{\kappa}_{r}$ and $\Omega$. The idea is to apply, on each cross-section $S$ of $H$, the strategy used in the isolated horizon framework to select a preferred permissible vector field $t_{o}^{a}$ :

i) Calculate the angular momentum $J_{S}$ defined by the axial Killing field $\varphi^{a}$. This provides us with a function $J(R)$ on the horizon $H$;

ii) Set

$$
\bar{\kappa}_{r}=\kappa_{o}(R):=\frac{R^{4}-4 G^{2} J^{2}}{2 R^{3} \sqrt{R^{4}+4 G^{2} J^{2}}} .
$$


This is achieved by solving for $d r / d R=2 R \bar{\kappa}_{o}(R)$, which determines $r$ and $N_{r}$; and, iii) choose $\Omega$ such that

$$
\Omega=\Omega_{o}(R):=\frac{2 G J}{R \sqrt{R^{4}+4 G^{2} J^{2}}} .
$$

This functional dependence of $\bar{\kappa}_{r}$ and $\Omega$ on $R$ and $J$ is exactly that of the Kerr family. That is, given a cross-section $S$, we choose $t_{o}^{a}$ which has the same effective surface gravity and angular velocity at that cross-section as the surface gravity and angular velocity that the time-translation Killing field has on the horizon of the Kerr solution with the same area and angular momentum. Our task now is to integrate eq. (5.12). For this, let us first recall the properties of the standard Smarr formula for the Kerr family:

$$
M(R, J):=2\left(\frac{\kappa_{o} a}{8 \pi G}+\Omega_{o} J\right)=\frac{\sqrt{R^{4}+4 G^{2} J^{2}}}{2 G R} .
$$

The function $M$ of two variables $R, J$ has the property that under arbitrary variations of the two parameters the first law, $\delta M=\left(\kappa_{o} / 8 \pi G\right) \delta a+\Omega \delta J$, is satisfied. Therefore, it follows that

$$
E^{t_{o}}(R):=M(R, J(R))
$$

satisfies the differential equation (5.12). Furthermore, it is the unique solution which reduces to the expression (5.13) in the case of spherical symmetry (when $J(R)=0$ identically).

This notion of horizon energy has some attractive properties. First, it depends only on geometrical fields on each cross section and the dependence is local. Yet, as noted in section $\mathrm{V} A$, thanks to the constraint part of Einstein's equations, changes in $E^{t_{o}}$ over finite regions $\Delta H$ of $H$ can be related to the expected fluxes:

$$
E_{S_{2}}^{t_{o}}-E_{S_{1}}^{t_{o}}=\mathcal{F}_{\text {grav }}^{\left(t_{o}\right)}+\mathcal{F}_{\text {matter }}^{\left(t_{o}\right)}
$$

where the flux of gravitational energy $\mathcal{F}_{\text {grav }}^{\left(t_{o}\right)}$ is local and positive definite (see (5.7)). (The gravitational angular momentum flux which, in general, has indeterminate sign vanishes due to axi-symmetry.) Finally, as mentioned in section III, Booth and Fairhurst have recently shown that this expression of the dynamical horizon energy emerges from a systematic Hamiltonian framework on space-times $\mathcal{M}$ with a dynamical horizon $H$ as inner boundary [22].

Note that, as a function of its angular momentum and area, each cross-section $S$ is assigned simply that $E_{S}^{t_{o}}$ which it would have in the Kerr family. Physically, this is a simple and attractive property. Furthermore, because of its close relation to the Kerr time translation, $\left.t_{o}\right|_{S}$ represents that 'time-translation for which the horizon is at rest at the instant $S^{\prime}$. Therefore, we will refer to $E^{\left(t_{o}\right)}$ as the mass function on the (axi-symmetric) dynamical horizon $H$ and set $E^{t_{o}}=M(R)$. (The overall strategy is the same as that used in the isolated horizon framework [7].) Thus, among the infinitely many first laws (5.8), there is a canonical one:

$$
d M=\frac{\bar{\kappa}_{o}}{8 \pi G} d a+\Omega_{o} d J .
$$

We conclude this section with a discussion of the possibility that a dynamical horizon can have an excess of angular momentum and violate the Kerr bound $J \leq G M^{2}$ and the possibility of extracting rotational energy from the black hole. ${ }^{7}$ In the Kerr solution, it is

\footnotetext{
${ }^{7}$ For the discussion that follows, it is convenient to note that in the Kerr family the limiting, extremal Kerr horizon results when $2 G J=R^{2}=2 G^{2} M^{2}$.
} 


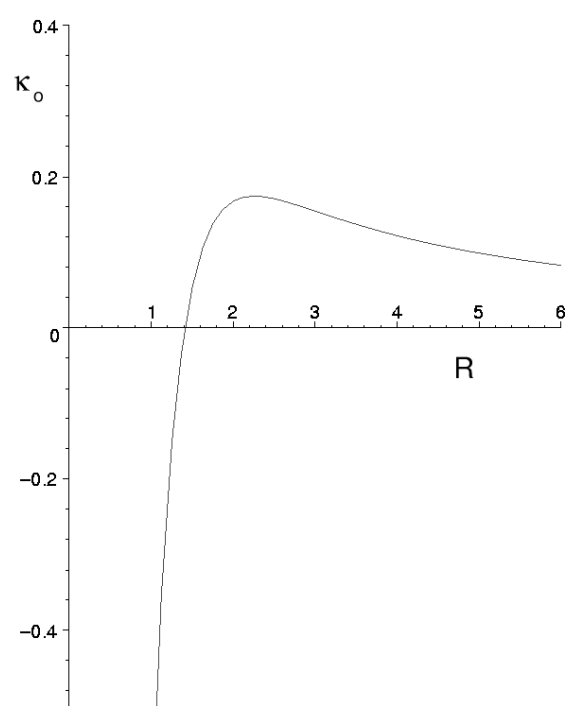

FIG. 2: A plot of the Kerr surface gravity $\kappa_{o}$ (from eq. (5.16)) as a function of $R$ (with $J G$ set equal to 1 for definiteness). The part $\kappa_{o}<0$ is the Kerr forbidden side while $\kappa_{o}>0$ is the Kerr allowed regime. Since $R$ increases monotonically with time, this graph shows that the dynamical horizon always evolves toward the Kerr allowed region under time evolution.

forbidden to violate the inequality $J \leq G M^{2}$. However, none of the equations we derived rule out the possibility that a dynamical horizon may be formed with a cross section $S$ on which the Kerr limit is violated, i.e., $2 J G>R^{2}$. On this $S$, we will have $\kappa_{o}<0$ (see eq. (5.16)) so that, with our prescription for constructing $t_{o}, \bar{\kappa}_{r}$ would be negative (whence $r$ would be a decreasing function of $R$ ). But the prescription for selecting $t_{o}^{a}$ still goes through and the dynamical horizon mass, given by:

$$
M(R)=\frac{\sqrt{R_{\Delta}^{4}+4 G^{2} J^{2}}}{2 G R_{\Delta}}
$$

which is well-defined, positive. Let us first consider the case when $T_{a b}$ vanishes on the horizon. Then, because of axi-symmetry, $J$ is constant. On the other hand, the area always increases. What happens to surface gravity? In the Kerr allowed region it is positive and in the Kerr forbidden region, negative. Are we driven toward the Kerr allowed region or further away from it? A simple calculation yields:

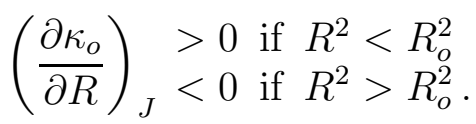

where $R_{o}^{2} \sim 5.085 J G$. This is also shown graphically in figure 2 as a plot of $\kappa_{o}(R)$ versus $R$ for a fixed value of $J$. Therefore, on the Kerr-forbidden side, under time evolution $R$ increases whence the surface gravity also increases, i.e., becomes less negative, and we are pushed toward the extremal point. When the radius increases so that $R^{2}>5.085 J G$, surface gravity starts decreasing but this is essentially irrelevant because we are now on the Kerr-allowed side where surface gravity is always positive (and tends to zero as area tends to infinity, keeping $J$ fixed). These considerations suggest that a black hole may well be formed in the Kerr-forbidden region and then settle down to a Kerr hole as time evolves. 
Since we put $T_{a b}=0$, this process can happen even in vacuum general relativity, e.g., in black hole mergers. At first this seems counter-intuitive because there are heuristic arguments which suggest that the black hole cannot radiate more angular momentum than energy, whence if it is initially formed with $J>G M^{2}$, it would not be able to settle down to a Kerr state in the distant future. However, what can happen is the following. Initially, one may have $J>G M^{2}$ but there may be energy trapped between the black hole and the 'peak of the potential' outside the horizon, which may fall in the black hole, increasing its mass significantly but keeping its angular momentum the same, thereby moving its state toward the Kerr allowed regime. Finally, in presence of matter, the flux $\mathcal{F}_{\text {matter }}^{\left(t_{o}\right)}$ need not be positive definite because if $\Omega \neq 0$, the vector field $t_{o}^{a}$ is space-like at the horizon. In this case, the in-falling matter could pour negative angular momentum into the black hole thereby decreasing its mass.

Thus, it is rather surprising that there is no obvious obstruction for a dynamical horizon to be first formed in the Kerr forbidden region and yet fulfill the physical expectation that the final, equilibrium state should be a Kerr horizon. We should emphasize however that the issue of whether this is compatible with solutions to the constraint equations on $H$ is yet to be analyzed. In physically interesting situations, there is a further very non-trivial restriction: one is interested only in those horizons which arise in the dynamical evolution of physically appropriate initial data on Cauchy surfaces. Nonetheless, the fact that there is no obvious obstruction suggests that the issue should be analyzed further.

We conclude with two remarks.

i. The cosmological constant: Our discussion can be generalized to the $\Lambda \neq 0$ case in a rather straightforward manner by replacing the current, Kerr expressions of $\kappa_{o}(R)$ and $\Omega_{o}(R)$ by those from Kerr-de Sitter and Kerr-anti de Sitter space-times.

ii. Physical relevance of $t_{o}^{a}$ : In this sub-section, we introduced a family of physically motivated vector fields $t_{o}^{a}$ and showed that the corresponding energy $E_{S}^{t_{o}}$ is determined by fields defined locally on $S$. However, this is by no means the only vector field with this property. To a certain extent, an analogy at null infinity is provided by the Bondi-Metzner-Sachs supertranslations: There is an infinite dimensional family of supertranslations, each associated with a local flux, a 2-sphere supermomentum integral, and a balance law [11]. Although the supermomenta and their fluxes do carry physical information, it is the 4-momentum and its flux that is most important physically and admits a direct and transparent interpretation. Similarly, on dynamical horizons, of all permissible vector fields $t^{a}$ leading to local energy expressions $E_{S}^{t}$, it is likely that $t_{o}^{a}$ would be the most relevant one from physical considerations. In particular, one expects that in the asymptotic future the dynamical horizon would tend to a Kerr isolated horizon $[7,26]$ and $E_{S}^{t_{o}}$ would tend to the mass of that Kerr space-time.

\section{Mechanics versus thermodynamics}

In stationary space-times - and more generally, in the isolated horizon framework - the horizon geometry is time independent and this in particular implies that the surface gravity $\kappa$ is constant on the horizon. In the physical process version of the first law, $d E=(\kappa / 8 \pi G) d a+$ $\Omega d J$, one considers transitions from a time independent state to a nearby time independent state. Conceptually, this is the same setting as in laws of equilibrium thermodynamics. As 
in the case of the first law of thermodynamics, the second term represents mechanical work done on the horizon while the first term does not; it is interpreted as the analog of the term $T d S$ representing the 'heat absorbed by the black hole'. The specific form of this term shows that, in infinitesimal processes involving black holes, the change in surface gravity can be ignored just as the change in the temperature is ignored in the transitions envisaged by the first law.

By contrast, in this paper we considered fully dynamical situations in which the horizon geometry can be very far from being stationary. We obtained two closely related results, the balance equation (5.7) and the integral generalization (5.9) of the first law. The first can be directly interpreted as a statement of conservation of energy, in that it describes how the energy of the dynamical horizon changes because of the influx of matter and gravitational waves. The second is an integral version of the first law of black hole mechanics because it tells us how the changes in the characteristics of the black hole - the area and the angular momentum - are correlated with changes in its energy.

Let us focus on the second. The angular momentum term can again be interpreted as mechanical work done on the black hole. What about the term representing the change in area? Is there again a close analogy with thermodynamics? To analyze this issue, we must consider fully non-equilibrium thermodynamical processes. Generically, the system does not have time to come to equilibrium in these processes and there is no canonical notion of its temperature. Therefore, while one can still interpret the difference $E_{2}-E_{1}-$ (work) as the heat absorbed by the system, in general there is no longer a clean split of this term in to a temperature part and a change in entropy part. If the process is such that the system remains close to equilibrium throughout the process, i.e., can be thought of as making continuous transitions between a series of equilibrium states, then the difference can be expressed as $\int T d S$, where the temperature $T$ varies slowly during the transition.

The situation on dynamical horizons is analogous. ${ }^{8}$ On general dynamical horizons, the time dependence can be strong, and physically one does not expect the term $E_{S_{2}}^{t_{o}}-E_{S_{1}}^{t_{o}}-$ (work) to admit a natural split into a temperature part and a change in entropy part. Indeed, if the horizon geometry is changing very rapidly, it cannot be considered to be in a nearequilibrium state whence it would be inappropriate to associate an instantaneous physical temperature to it. How does one reconcile this with the fact that in eq. (5.6) the difference is expressed as $\int \bar{\kappa}_{r} d a$ ? The resolution lies in the fact that $\bar{\kappa}_{r}$ is only the effective surface gravity. More precisely, in striking contrast to what happens in equilibrium configurations represented by isolated horizons, $\bar{\kappa}_{r}$ does not have the geometrical interpretation of surface gravity; as shown in section VI, it can only be interpreted as the 2-sphere average of a geometrical surface gravity associated with certain vector fields on $H$. This is a reflection of the limitation that, in highly dynamical situations, $\bar{\kappa}_{r}$ should not have a direct interpretation of instantaneous, physical temperature.

One would expect such an interpretation to be meaningful only if the time dependence is weak, i.e., on weakly-dynamical horizons which can be regarded as perturbations of isolated horizons. In this case, the geometrical surface gravity [22] (see section VI) would be approximately constant and thus approximately equal its average, $\bar{\kappa}_{r}$. In this situation, one can regard the horizon as making continuous transitions from one equilibrium state to another and then the geometrical surface gravity appears to be a good analog of the (slowly

\footnotetext{
8 This point was emphasized by S. Fairhurst at the Black hole IV workshop, held at Honey Harbor, Canada in May 2003 and at the Penn State Decennial conference in June 2003.
} 
varying) temperature. In these situations, the dynamical first law (5.6) can be simplified by keeping terms only up to second order in perturbations away from isolation [27]. In this approximation, $E_{S_{2}}^{t_{o}}-E_{S_{1}}^{t_{o}}-$ (work) can be interpreted as ' $\int \kappa d a$ ' where $\kappa$ has a geometrical interpretation as surface gravity. Hence, the simplified version of (5.9) can be regarded as the integral version of the first law of black hole thermodynamics.

These considerations have interesting implications to the notion of black hole entropy in dynamical situations. Because the horizon area increases in dynamical processes, in view of the second law of thermodynamics, it is tempting to identify a suitable multiple the horizon area with entropy. In time independent situations, this interpretation is confirmed also by the first law (1.1) because the term $(\kappa / 8 \pi G) d a$ is analogous to the term $T d S$ in the first law of thermodynamics. The above discussion suggests that the interpretation should continue to be valid also on weakly-dynamical horizons. It is therefore interesting to analyze if the black hole entropy derivations based on counting of micro-states, such as those of [28], can be extended to this case. For highly dynamical situations, on the other hand, the situation is less clear. In the case of event horizons, for example, one would not expect this formula for entropy to be meaningful because, as mentioned in section I, an event horizon can be formed and grow in a flat space region in anticipation of a future gravitational collapse. It is difficult to imagine how a quasi-local counting of micro-states can account for this phenomenon. The case of highly dynamical horizons falls in-between. On the one hand, the case for identification of entropy with a multiple of area is now much weaker than on weakly-dynamical horizons for reasons discussed above. On the other hand, somewhat surprisingly, the term $\left(r_{2}-r_{1}\right) / 2 G$ of $(5.9)$ can be expressed as $(1 / 8 \pi G) \int \bar{\kappa}_{r} d a$ even in the fully dynamical regime. Furthermore, since the growth of area $i s$ related to the energy flux across the horizon, it may well be possible that a quasi-local counting arguments along the lines of [28] can be constructed in this case.

\section{TRANSITION TO EQUILIBRIUM}

The conventions we used in all the calculations up to this stage are well-suited to the space-like character of $H$. When $H$ reaches equilibrium, there is no longer a flux of matter or gravitational energy across it whence (with appropriate normalization) the shear and the matter flux vanishes. Eq. (2.1) now implies that the horizon must become null. Furthermore, since the expansion $\Theta_{(\ell)}$ vanishes, it is a non-expanding horizon in the sense of $[5,6]$. The goal of this section is to analyze the transition from a dynamical horizon to a non-expanding one. In most physical situations, because of back-scattering, one can expect the equilibrium to be reached only asymptotically, i.e., in the infinite future. (For exceptions, see the examples discussed in Appendix A.) However, as we will see, the case of asymptotic equilibrium is technically simpler but the subtleties involved in the matching at a finite time are more instructive.

\section{A. The non-expanding horizon}

Let us then consider a 3-manifold $M$, topologically $S^{2} \times R$ which is the union of a dynamical horizon $H$ and a non-expanding horizon $\Delta$ (see figure 1). Thus, $H$ is space-like and foliated by a family of marginally trapped surfaces $S$, while $\Delta$ is null. Denote the past boundary of $\Delta$ by $S_{0}$ which will be assumed to be the (uniform) future limit of the cross- 
sections $S$ of $H$. We will assume that: i) the space-time metric $g_{a b}$ is $C^{k}$ for some $k \geq 2$; ii) $M$ is a $C^{k+1}$ sub-manifold; and iii) the pull-back $q_{a b}$ to $M$ of $g_{a b}$ admits an axial Killing field $\varphi^{a}$.

Let us first consider $\Delta$. It has the property that the expansion of any of its null normals vanishes. However, to extract physics, one needs to endow it with an additional structure. Since $\Delta$ is null, it follows that $\bar{\ell}^{a} \nabla_{a} \bar{\ell}^{b}=\kappa_{\bar{\ell}} \bar{\ell}^{b}$ for any of its null normals $\bar{\ell}^{a}$. The extra structure consists of an equivalence class $[\bar{\ell}]$ of null normals whose acceleration, or surface gravity $\kappa_{\bar{\ell}}$, is constant on $\Delta$, where $\bar{\ell}^{a}$ and $\left(\bar{\ell}^{\prime}\right)^{a}$ are equivalent if and only if $\left(\bar{\ell}^{\prime}\right)^{a}=c \bar{\ell}^{a}$ where $c$ is a constant on $\Delta$. Such a choice can always be made but it is far from being unique [5]. (The freedom is exhibited in section VIB.) The pair $(\Delta,[\bar{\ell}]$ ), where $[\bar{\ell}]$ satisfies this condition, defines a weakly isolated horizon.

On weakly isolated horizons, one can introduce the notion of energy and angular momentum such that the zeroth and the first laws of black hole mechanics hold. Not only is the angular momentum $J_{\Delta}^{\varphi}$ conserved as one would expect because of 'isolation', but its value turns out to be independent of the specific choice of $[\bar{\ell}]$ made in the transition from the non-expanding horizon to the weakly isolated one. To define energy, one needs to introduce 'permissible' vector fields $t^{a}[6,7]$ and there is an infinite family of these. However, on any weakly isolated horizon $(\Delta,[\bar{\ell}])$, one can choose a canonical one, $t_{0}^{a}=\bar{\ell}_{0}^{a}-\Omega_{0} \varphi^{a}$, such that the surface gravity of $\bar{\ell}_{0}^{a}$ and the angular velocity $\Omega_{0}$ are determined by the area $a_{\Delta}$ and the angular momentum $J_{\Delta}^{\varphi}$ exactly as on the Kerr horizon. Again, when this choice is made, the value of $E_{\Delta}^{t_{0}}$ is independent of the specific equivalence class $[\bar{\ell}]$ chosen in the transition from non-expanding to weakly isolated horizons. In this sense, the angular momentum and the mass are properties of non-expanding horizons themselves, although in the intermediate stages in the calculation one has to pick a weakly isolated horizon structure.

However, the vector fields $t_{0}^{a}$ do vary with the choice of admissible $[\bar{\ell}]$; they all just happen to lead to the same value of energy. In our case, $\Delta$ is the limit of a dynamical horizon $H$, whence it is natural to pick that $t_{0}^{a}$ on $\Delta$ which arises as the limit of the canonical vector field $t_{o}^{a}$ on $H$ (introduced in Section VB). This will in turn fix the weakly isolated horizon structure on $\Delta$ uniquely.

\section{B. An intermediate construction on the dynamical horizon}

To carry out the matching, we need to introduce some additional structure on $H$. This structure will enable us to take the limits to $S_{0}$ and also clarify the meaning of the "effective surface gravity' introduced in section V A.

Throughout our calculations so far, we used the unit normal $\widehat{\tau}^{a}$ to $H$ and the unit normal $\widehat{r}^{a}$ within $H$ to the cross-sections $S$. In particular the null vectors $\ell^{a}, n^{a}$, which played a dominant role throughout, are the sum and differences of these normals. This structure is well-suited to the space-like character of $H$. However, when we consider the transition, because $\Delta$ is null, these fields either diverge or vanish as we approach $S_{0}$. Therefore, to study the limit to equilibrium, we need to rescale these fields suitably.

With this goal in mind, let us begin by introducing a smooth vector field $\bar{V}^{a}$ which can be regarded as a smooth (space-like) extension to $H$ of a suitable $\bar{\ell}^{a}$ on $\Delta$. As discussed in section VIA, we would like $\bar{\ell}^{a}$ on $\Delta$ such that its surface gravity $\kappa_{\bar{\ell}}$ equals the Kerr value $\kappa_{o}\left(a_{\Delta}, J_{\Delta}\right)$. Such vector fields always exist and the freedom in their choice is given by [5]:

$$
\bar{\ell}^{\prime a}=\left(1+A e^{-\kappa_{o} v}\right) \bar{\ell}^{a}
$$


if $\kappa_{o} \neq 0$ and

$$
\bar{\ell}^{\prime a}=B \bar{\ell}^{a}
$$

$\kappa_{o}=0$, where $v$ is the affine parameter of $\bar{\ell}^{a}$ and the functions $A, B$ on $\Delta$ satisfy: $\mathcal{L}_{\bar{\ell}} A=$ $0, \mathcal{L}_{\bar{\ell}} B=0$ and $(1+A)>0, B>0$. On $H$, it is natural to require that $\bar{V}^{a}$ to be parallel to $\widehat{r}^{a}$ and map cross-sections of $H$ to themselves. This condition determines $\bar{V}^{a}$ up to a rescaling by a function of $R$. Given any one of these $\bar{V}^{a}$, one can use the freedom available in the choice of $\bar{\ell}^{a}$ on $\Delta$ to pick one that will match smoothly with $V^{a}$ on $H$.

Fix one of these vector fields $\bar{V}^{a}$ and define multiples $\bar{n}^{a}$ and $\bar{\ell}^{a}$ of $\ell^{a}$ and $n^{a}$ via: $V^{a} \bar{n}_{a}=$ -2 and $\bar{\ell}^{a} \bar{n}_{a}=-2$. By construction, the barred fields are smooth on $M$. Using the fact that $\bar{V}^{a}$ is parallel to $\widehat{r}^{a}$, we have: $\bar{V}^{a}=\bar{\ell}^{a}-b^{2} \bar{n}^{a}$ for some smooth function $b$. Since $\bar{V}^{a} \bar{V}_{a}=4 b^{2}$, and since $\bar{V}^{a}$ becomes null at $S_{0}$, it follows that $b$ tends to zero as we approach $S_{0}$ along $H$ and stays zero on $\Delta$. It is easy to check that the null fields $\bar{\ell}^{a}$ and $\bar{n}^{a}$ are related to our original $\ell^{a}$ and $n^{a}$ through $\bar{\ell}^{a}=b \ell^{a}$ and $\bar{n}^{a}=b^{-1} n^{a}$. Since barred fields are smooth across $S_{0}$, it follows that $\ell^{a}$ diverges and $n^{a}$ tends to zero as we approach $S_{0}$. Since $R$ is constant on $\Delta$, we also know that $d R$ and hence $N_{R}$ and $N_{r}$ all go to zero as we approach $S_{0}$ along $H$. In section VIC we will show that they do so at the 'same rate' as $b$. Since $\bar{\ell}^{a}=b \ell^{a}$, this will establish that the field $N_{r} \ell^{a}$ used in the construction of $t_{o}^{a}$ on $H$ admits a limit to $S_{0}$. (Throughout this discussion, $N_{r}$ will be the lapse featuring in the expression of the canonical vector field $t_{o}^{a}=N_{r} \ell^{a}-\Omega(R) \varphi^{a}$ on $H$.)

\section{Matching of physical quantities}

Let us begin by showing that the limit of the angular momentum $J_{S}^{\varphi}$ of cross-sections $S$ of $H$ equals the angular momentum $J_{\Delta}^{\varphi}$ defined on $\Delta$. The angular momentum on any cross-section $S$ of $H$ is given by (4.2):

$$
J_{S}^{\varphi}=-\frac{1}{8 \pi G} \oint_{S} K_{a b} \varphi^{a} \widehat{r}^{b} d^{2} V
$$

Using the definition of $K_{a b}$ and expanding the vectors $\widehat{\tau}^{a}$ and $\widehat{r}^{a}$ in terms of $\bar{\ell}^{a}$ and $\bar{n}^{a}$ which are well-defined on all of $M$, we can rewrite the integrand as

$$
K_{a b} \varphi^{a} \widehat{r}^{b}=\bar{\omega}_{a} \varphi^{a}-\varphi^{a} \nabla_{a} \ln b,
$$

where $\bar{\omega}_{a}$ is the pull-back to $M$ of $-\frac{1}{2} \bar{n}_{b} \nabla_{a} \bar{\ell}^{b}$. Hence, using the fact that $\varphi^{a}$ is divergence-free on $S$, we obtain:

$$
J_{S}^{\varphi}=-\frac{1}{8 \pi G} \oint_{S} \bar{\omega}_{a} \varphi^{a} d^{2} V
$$

Since the integrand is smooth, the future limit of $J_{S}^{\varphi}$ as we approach $S_{0}$ is obtained by just evaluating the right side on $S_{0}$. This is precisely the angular momentum $J_{\Delta}^{\varphi}$ associated with the non-expanding horizon [7]. Thus, the angular momentum defined on $H$ matches smoothly with that defined on $\Delta$.

Let us consider the energy. $E_{S}^{t_{o}}$ on any cross-section $S$ of $H$ and $E_{\Delta}^{t_{0}}$ on $\Delta$ are functions of the angular momentum and area and their form is determined by the functional dependence on area and angular momentum of the mass function in Kerr metrics. Since the area and angular momentum match smoothly, we are immediately led to the conclusion that $E_{S}^{t_{o}}$ matches smoothly with $E_{\Delta}^{t_{0}}$ as $S$ approaches $S_{0}$. 
Finally, we will show that the vector field $t_{o}^{a}$ on $H$ matches smoothly to a 'permissible' vector field $t_{0}^{a}$ on $\Delta$. While these fields do not have a direct physical significance as far as final results are concerned, since energy $E_{S}^{t_{o}}$ on $H$ is associated with the vector field $t_{o}^{a}$ and $E_{\Delta}^{t_{0}}$ is associated with $t_{0}^{a}$, for conceptual completeness, we need to verify that the two vector fields match at $S_{0}$. To explore the relation between the two, the key idea is to use the property

$$
\mathcal{L}_{\bar{V}} \tilde{\epsilon}_{a b}=-\frac{1}{2} b^{2} \Theta_{(\bar{n})} \tilde{\epsilon}_{a b}
$$

on $H$, where $\tilde{\epsilon}_{a b}$ is the intrinsic area 2 -form on the cross-sections $S$. Integrating this equation on any one cross-section, using $a=4 \pi R^{2}$, and the fact that $d r / d R=2 R \bar{\kappa}_{r}$, we obtain:

$$
d r=-\left[\frac{R \bar{\kappa}_{r}}{4 \pi R} \oint_{S} b^{2} \Theta_{(\bar{n})} d^{2} V\right] d v
$$

on $H$ where $v$ is the affine parameter along $\bar{V}^{a}$ which takes constant values on cross-sections on $H$. Since $N_{r}=|d r|$ and $1 / 2 b=|d v|$, it now follows that

$$
\frac{N_{r}}{b}+\frac{R \bar{\kappa}_{r}}{8 \pi R b^{2}} \oint_{S} b^{2} \Theta_{(\bar{n})} d^{2} V=0
$$

Now, the second term admits a smooth limit to $S_{0}$, whence the limit of $N_{r} / b$ is also welldefined. (Furthermore, since $b$ is positive and $\Theta_{(\bar{n})}$ negative on $H$, it follows that the limit is nowhere zero.) This in turn implies that $t_{o}^{a}=N_{r} \ell^{a}-\Omega_{o} \varphi^{a}$ also admits a well-defined limit to $S_{0}$.

Our final task is to show that this vector field admits a smooth extension to a 'permissible' vector field $t_{0}^{a}=\bar{\ell}_{0}^{a}-\Omega_{0} \varphi^{a}$ on $\Delta$, where $\bar{\ell}_{0}^{a}$ has Kerr surface gravity $\kappa_{o}$ and $\Omega_{0}$ is the Kerr angular velocity. Since $N_{r} \ell^{a}$ has a smooth, nowhere vanishing limit, one can always use the rescaling freedom in (6.1) and (6.2) to choose the desired $\bar{\ell}_{0}^{a}$. The matching of $\Omega_{0}$ is guaranteed simply by setting $\Omega_{0}=\Omega_{o}$. Thus, there is a smooth vector field on $M$ which is a 'permissible' evolution field on $\Delta$ and agrees with the 'canonical' $t_{0}^{a}$ on $H$. Furthermore, this construction provides us with a 'canonical' weakly isolated horizon structure $\left[\bar{\ell}_{0}\right]$ on $\Delta$.

Thus, the results for transition to equilibrium at a finite time can be summarized as follows. Assuming that $M=H \cup \Delta$ is $C^{k+1}$, and the rotational vector field $\varphi^{a}$ on $M$ is $C^{k}$, one finds that: i) There is a $C^{k}$ matching of the angular momentum $J_{S}^{\varphi}$ on $H$ with $J_{\Delta}^{\varphi}$ on $\Delta$; ii) there is a unique weakly isolated horizon structure $\left[\bar{\ell}_{0}\right]$ on $H$ such that the canonically chosen vector field $t_{o}^{a}$ on $H$ has a $C^{k}$ matching with $t_{0}^{a}$ on $\Delta$; and, iii) the corresponding energies $E_{S}^{t_{o}}$ and $E_{\Delta}^{t_{0}}$ match in a $C^{k}$ manner.

If the horizon reaches equilibrium only asymptotically, $M$ is space-like everywhere and becomes null only asymptotically. In this case, we can just use the structure we already have on $H$ from sections III to $\mathrm{V}$ to describe dynamics. The discussion of this section implies that the asymptotic state should be identified with the weakly isolated horizon $(\Delta,[\ell])$ where the equivalence class $[\ell]$ is determined by $t_{o}^{a}$ on $H$. With this identification, the asymptotic limit is reached smoothly.

We conclude with two remarks.

i. surface gravity: Following Booth and Fairhurst [27], one can define surface gravity of $\bar{V}$ on $H$

$$
\kappa_{\bar{V}}:=-\frac{1}{2} \bar{n}_{b} \bar{V}^{a} \nabla_{a} \bar{V}^{b}
$$


which matches smoothly with the surface gravity $\kappa_{o}$ on $\Delta$. Furthermore, one can use it to restrict the freedom in the choice of $\bar{V}^{a}$ considerably by requiring that the 2-surface average of $\kappa_{\bar{V}}$ be the effective surface gravity $\bar{\kappa}_{r}=\kappa_{o}$ introduced in section V A:

$$
\oint_{S} \kappa_{V} d^{2} V=\kappa_{o}(R, J(R)) a_{S}
$$

where $a_{S}$ is the area of the cross-section $S$. Then the only remaining freedom is that of a constant:

$$
\bar{V}^{a} \longrightarrow\left(1+c \exp -\frac{1}{2} \int \kappa_{o} d v\right) \bar{V}^{a}
$$

where $v$ is the affine parameter along $\bar{V}$ and $c$ a constant. In the case when equilibrium is reached only asymptotically, all these vector fields tend to the same null vector.

ii. Slowly evolving horizons: Heuristically, one expects that near the transition surface $S_{0}, H$ would become weakly dynamical and can be regarded as a perturbed, non-expanding horizon. However, strictly, weakly dynamical horizons can and should be defined in their own right because, as Appendix A shows, a dynamical horizon can have strong time dependence arbitrarily close to the transition surface. A notion of 'slowly evolving horizons' has already been introduced in [27] and our introduction of the vector field $\bar{V}^{a}$ was motivated by that analysis. However, in the calculation of energy, [27] uses only a space-like vector field analogous to $\bar{V}^{a}$, in place of our null vector $N_{r} \ell^{a}$. Hence that analysis is based only on the momentum constraint (2.5); the Hamiltonian constraint (2.4) plays no role. This is probably because $\bar{V}^{a}=\bar{\ell}^{a}-b^{2} \bar{n}^{a}$ and, in the leading order approximation studied in [27], the $\bar{n}^{a}$ term can be neglected. However, the detailed relation between our discussion of passage to equilibrium and that discussion of slowly evolving horizons is yet to be understood.

\section{DISCUSSION}

Let us begin with a brief summary. A dynamical horizon is a space-like 3-manifold, foliated by 2-dimensional closed, marginally trapped surfaces $S$ (called cross-sections) on which the expansion of the inward null normal is negative. While the definition is so simple and conditions in it appear to be quite weak, dynamical horizons turned out to have remarkable properties. Specifically, we were able to: i) propose a definition of the flux of gravitational energy falling across a portion $\Delta H$ of $H$ bounded by two cross-sections and show that it is local, manifestly positive and gauge invariant (i.e. does not depend on any structure that is not intrinsic to the problem); ii) provide a detailed area balance law relating the change in the area of $H$ to the flux of energy across it; iii) show that the cross-sections $S$ of $H$ have the topology $S^{2}$ if the cosmological constant $\Lambda$ is positive and of $S^{2}$ or $T^{2}$ if it is zero. The $T^{2}$ case is degenerate in the sense that matter as well as gravitational energy fluxes vanish, the intrinsic metric on each cross section is flat, the shear of the (expansion-free) null normal $\ell^{a}$ vanishes and the derivatives of the expansion along both null normals vanish; iv) introduce the notion of angular momentum associated with each cross-section and of the flux across portions $\Delta H$ of the horizon bounded by two cross-sections; v) provide an integral generalization of the first law of black hole mechanics to fully dynamical situations; vi) For axi-symmetric horizons, give a prescription to find a vector field $t_{o}^{a}$ on $H$ and introduce a notion of energy $E_{S}^{t_{o}}$ for each cross section $S$ such that an easily interpretable balance law 
holds: if the portion $\Delta H$ of the horizon is bounded by $S_{2}$ and $S_{1}$, then $E_{S_{2}}^{t_{o}}-E_{S_{1}}^{t_{o}}=\mathcal{F}_{\Delta H}^{t_{o}}$, where $E_{S}^{t_{o}}$ and the flux $\mathcal{F}_{\Delta H}^{t_{o}}$ are both local and have physically attractive properties; and, vii) analyze in detail the passage to equilibrium during which a dynamical horizon becomes a weakly isolated one.

Let us highlight a few features of the framework and the results:

- i) Our analysis is not motivated by nor directly related to the issue of finding quasilocal mass in general relativity. Our results pertain to very special 2-surfaces - the cross-sections of dynamical horizons - and cannot be applied in more general context. Nonetheless, there is a general expectation that a dynamical black hole space-time would admit a large number of dynamical horizons and it is somewhat surprising that they all have such nice properties.

- ii) While the definition of the dynamical horizons assumes that the expansion $\Theta_{(n)}$ of the inward pointing null normal should be negative, most of the detailed results go through also in the case when $\Theta_{(n)} \leq 0$. Under the stronger assumption the area monotonically increases. Under the weaker assumption we only know that it cannot decrease, but the balance laws and the generalization of black hole mechanics still goes through. If $\Theta_{(n)} \geq 0$, we are in the white hole situation in which the results again apply with appropriate sign changes.

- iii) The preferred vector field $t_{o}$ has been chosen with the physical problems of black hole formation and coalescence in mind. In particular, the energy $E_{S}^{t_{o}}$ associated with a cross-section $S$ is precisely the mass of the Kerr space-time which has the same area and angular momentum as $S$; one regards $S$ as being 'instantaneously Kerr'. The surprising fact is that even in the fully dynamical regime, the difference between energies associated with two cross-sections is given by a local, geometrically defined flux. In the non-rotating case, $E^{t_{o}}$ reduces to the Hawking mass. But in the rotating case, the Kerr mass seems to be a better measure of the physical energy of the horizon (associated with the vector field $t_{o}^{a}$ ).

- iv) What is the situation in higher dimensions? Since our results stemmed from the constraint part of Einstein's equations in the metric variables, the method is directly applicable also in higher dimensions. But the form of results would be different. In particular, since the topological restriction made a crucial use of the Gauss-Bonnet theorem, it will not go through; since the black hole uniqueness theorem fails, there will be many distinct preferred vector fields $t_{o}^{a}$ and the most convenient choice will be dictated by the isolated horizon to which the dynamical one settles down to; and, some of the equations may now acquire Weyl tensor terms.

Finally, these results open up new avenues for further research in numerical, mathematical and quantum relativity. We will conclude by pointing out some of these.

Numerical and mathematical relativity: In a gravitational collapse or a black hole merger, one expects the dynamical horizon in the distant future to asymptotically approach a weakly isolated horizon. Can one establish that this expectation correct? If so, what can one say about the rate of approach? While this issue can be studied analytically, numerical simulations provide an ideal setting to analyze it because the world tube of apparent horizons arises there naturally and provides the dynamical 
horizon. There exists a simple, local characterization of the Kerr isolated horizon [26]. Under what conditions is one guaranteed that the asymptotic isolated horizon is the Kerr horizon? On an isolated horizon one can define multipoles invariantly [29] and the definition can be carried over to each cross-section of the dynamical horizon. What can one say about the rate of change of these multipoles? For example, from the knowledge of the horizon quadrupole and its relation to the Kerr quadrupole, can one gain insight in to the maximum amount of energy that can be emitted in gravitational radiation? Is the quasi-normal ringing of the final black hole coded in the rate of change of the horizon multipoles, as was suggested by somewhat heuristic considerations in the early numerical simulations [30] of non-rotating black holes?

Geometric analysis: Since $H$ is space-like, one can consider the standard initial value problem on it. Can one characterize the solutions to the constraint equations such that $\left(H, q_{a b}, K_{a b}\right)$ is a dynamical horizon? (It is trivial to check that the data cannot be time symmetric (i.e. $K_{a b}$ cannot be zero on $H$ ) but one could consider the constant mean curvature case.) A full characterization would provide a complete control on the geometry of the world tube of apparent horizons that will emerge in all possible numerical simulations! One can further ask: Can one isolate the freely specifiable data in a useful way? Are these naturally related to the freely specifiable data on weakly isolated horizons [5]? In the spherically symmetric case, these issues are straightforward to address and an essentially complete solution is known. It would be very interesting to answer these questions in the axi-symmetric case.

Another potential application is to the proof of Penrose inequalities which say that the total (ADM) mass of space-time must be greater than half the radius of the apparent horizon on any Cauchy slice. In the time symmetric case (i.e., when the extrinsic curvature on the Cauchy slice vanishes) this conjecture was recently proved by Huisken and Ilmamen, and Bray [14]. Our analysis provides two flows which may be potentially useful to extend the analysis beyond the time-symmetric case. The first is associated with the Hawking mass and was discussed in section III A: Eq. (3.25) shows that the Hawking mass increases monotonically along a dynamical horizon. Furthermore, one expects that the dynamical horizon would settle down to a weakly isolated horizon in the future. For isolated horizons which extend all the way to $i^{+}$, under certain regularity conditions, the horizon mass is the future limit of the Bondi mass [3]. Thus, using our flow, one should be able to prove a stronger version of the Penrose inequality where the ADM mass is replaced by the future limit of the Bondi mass. The second flow is associated with the Kerr mass and was discussed in section V. In the non-rotating case, this is the same as the first flow. But in the rotating case, the Kerr mass is greater than the Hawking mass whence it would provide a further strengthening of the Penrose inequality. Note that the Kerr mass increases monotonically only if one begins with a cross-section $S$ of $H$ on which $2 G J<R^{2}$. But if we initially violate this condition, the flow drives the system towards satisfaction of this inequality.

Quantum relativity: As the vast mathematical literature on black hole mechanics shows, the infinitesimal version (1.1) of the first law has had a deep conceptual influence. The finite version (5.9) may have similar ramifications in non-equilibrium situations. The Hamiltonian framework of Booth and Fairhurst [22] could be used as a point of departure for describing quantum black holes beyond equilibrium situations. Can one, 
in particular, extend the non-perturbative quantization of isolated horizons of $[28,29]$ to describe quantum, dynamical horizons? To calculate their entropy? To naturally incorporate back reaction in the Hawking process?

\section{Acknowledgements:}

We would like to thank Ivan Booth, Stephen Fairhurst, Sean Hayward, Jerzy Lewandowski and Tomasz Pawlowski for stimulating discussions and Lars Andersson, Chris Beetle, Bobby Beig, Matt Choptuik, Piotr Chrusciel, Sergio Dain, Sam Finn, Eanna Flanagan, Greg Galloway, Jim Hartle, Gary Horowitz, Gerhard Huisken, Tom Ilmanen, Jim Isenberg, Pablo Laguna, Luis Lehner, Bernd Schmidt, Walter Simon, Bill Unruh and Jeff Winicour for interesting comments and questions. This work was supported in part by the National Science Foundation grants PHY-0090091, PHY99-07949, the National Science Foundation Cooperative Agreement PHY-0114375, the Eberly research funds of Penn State and the Albert Einstein Institut.

\section{APPENDIX A: EXAMPLES: THE VAIDYA SOLUTIONS}

The Vaidya metrics provide simple, explicit examples of dynamical horizons. Furthermore, when the flux of the null matter field vanishes, one obtains an isolated horizon. Therefore, the metrics also provide explicit examples of the transition from the dynamical to isolated horizons discussed in section VI. In section A 1 we describe the SchwarzschildVaidya dynamical horizon and in section A 2, we include the cosmological constant.

\section{The Schwarzschild-Vaidya metrics}

In the ingoing Eddington-Finkelstein coordinates $(v, r, \theta, \phi)$ the 4-metric is given by

$$
g_{a b}=-\left(1-\frac{2 G M(v)}{r}\right) \nabla_{a} v \nabla_{b} v+2 \nabla_{(a} v \nabla_{b)} r+r^{2}\left(\nabla_{a} \theta \nabla_{b} \theta+\sin ^{2} \theta \nabla_{a} \phi \nabla_{b} \phi\right)
$$

where $M(v)$ is any smooth non-decreasing function of $v$. This is a solution of Einstein's equations with zero cosmological constant, the stress-energy tensor $T_{a b}$ being given by

$$
T_{a b}=\frac{\dot{M}(v)}{4 \pi r^{2}} \nabla_{a} v \nabla_{b} v
$$

where $\dot{M}=d M / d v$. Clearly, $T_{a b}$ satisfies the dominant energy condition if $\dot{M} \geq 0$ and vanishes if and only if $\dot{M}=0$.

Let us focus our attention on the metric 2-spheres given by $v=$ const, $r=$ const. The outgoing and ingoing null normals to these 2-spheres can be taken to be, respectively,

$$
\bar{\ell}^{a}=\left(\frac{\partial}{\partial v}\right)^{a}+\frac{1}{2}\left(1-\frac{2 G M}{r}\right)\left(\frac{\partial}{\partial r}\right)^{a} \quad \text { and } \quad \bar{n}^{a}=-2\left(\frac{\partial}{\partial r}\right)^{a}
$$

(so that $\bar{\ell}^{a} \bar{n}_{a}=-2$ as in the main text). The expansion of the outgoing null normal $\bar{\ell}^{a}$ is given by:

$$
\Theta_{(\bar{\ell})}=\frac{r-2 G M(v)}{r^{2}} .
$$


Thus, the only spherically symmetric marginally trapped surfaces are the 2 -spheres $v=$ const and $r=2 G M(v)$. The question is if these surfaces are cross-sections of a dynamical horizon. On each of these surfaces, the expansion of the ingoing normal $n^{a}$ is negative, $\Theta_{(\bar{n})}=-4 / r$. Furthermore, at the marginally trapped surfaces, $\mathcal{L}_{\bar{n}} \theta_{(\bar{\ell})}=-2 / r^{2}<0$. Because of spherical symmetry the shear of $\bar{\ell}^{a}$ (and $\bar{n}^{a}$ ) vanishes identically. Finally, $T_{a b} \bar{\ell}^{a} \bar{\ell}^{b}>0$ if and only if $\dot{M}>0$. Hence it follows from our general discussion in section II A (see equation (2.1)) that the surface $H$ given by

$$
r=2 G M(v) \quad \text { with } \quad \dot{M}>0
$$

is a dynamical horizon. When $\dot{M}$ vanishes, the surface $r=2 G M(v)$ becomes null and a non-expanding horizon (which is, in fact, an isolated horizon). The full surface $r=2 G M(v)$ is a future outer trapping horizon (FOTH) of Hayward's [15].

The null normals $\bar{\ell}^{a}$ and $\bar{n}^{a}$ are well suited for studying the approach to equilibrium, i.e., the transition from the dynamical to the isolated horizon discussed in section VI. The interpolating vector field $\bar{V}^{a}$ is now given by:

$$
\bar{V}^{a}=\left(\frac{\partial}{\partial v}\right)^{a}+2 G \dot{M}\left(\frac{\partial}{\partial r}\right)^{a} \equiv \bar{\ell}^{a}-G \dot{M} \bar{n}^{a}
$$

so that $b^{2}=G \dot{M}$. The degree of smoothness of the matching between the isolated and the dynamical horizons is dictated by differentiability of $\dot{M}$ at $S_{0}$. Thus, if $\dot{M}$ is $C^{k}$ on $S_{0}$, physical fields will match in a $C^{k}$ fashion. Finally, because of spherical symmetry the surface gravity $\kappa_{\bar{V}}$ of $\bar{V}^{a}$ is constant on each cross-section and is given by $1 / 2 R(v)$; the canonical vector field $t_{0}^{a}=(\partial / \partial v)^{a}$ on $\Delta$ matches smoothly with the canonical vector field $t_{o}^{a}=(\partial / \partial v)^{a}$ on $H$; the angular momentum vanishes; and, the horizon mass is given by $M(v)$.

To study the structure of $H$ by itself, as in the main body of the paper, a different normalization of null vectors is more convenient. For completeness, we list all the relevant vector fields:

$$
\begin{aligned}
\hat{\tau}_{a}=\frac{1}{2 \sqrt{G \dot{M}}} \nabla_{a} r-\sqrt{G \dot{M}} \nabla_{a} v & \hat{r}_{a}=\frac{1}{2 \sqrt{G \dot{M}}} \nabla_{a} r+\sqrt{G \dot{M}} \nabla_{a} v \\
n_{a}=-2 \sqrt{G \dot{M}} \nabla_{a} v & \ell_{a}=\frac{1}{\sqrt{G \dot{M}}} \nabla_{a} r \\
\hat{r}^{a}=\frac{1}{2 \sqrt{G \dot{M}}}\left(\frac{\partial}{\partial v}\right)^{a}+\sqrt{G \dot{M}}\left(\frac{\partial}{\partial r}\right)^{a} & \hat{\tau}^{a}=\frac{1}{2 \sqrt{G \dot{M}}}\left(\frac{\partial}{\partial v}\right)^{a}-\sqrt{G \dot{M}}\left(\frac{\partial}{\partial r}\right)^{a} \\
n^{a}=-2 \sqrt{G \dot{M}}\left(\frac{\partial}{\partial r}\right)^{a} & \ell^{a}=\frac{1}{\sqrt{G \dot{M}}}\left(\frac{\partial}{\partial v}\right)^{a} .
\end{aligned}
$$

Let us take $(r, \theta, \phi)$ as coordinates on the dynamical horizon. The radial coordinate $r$ is also the area coordinate $R$ in this case, whence $N_{R}$ and $\bar{\kappa}_{R}$ of the main text will be denoted just by $N_{r}$ and $\bar{\kappa}_{r}$ respectively. $N_{r}$ is given simply by

$$
N_{r}=\sqrt{G \dot{M}} .
$$

Therefore, the matter flux is

$$
\mathcal{F}_{\text {matter }}^{(r)}=\int_{\Delta H} N_{r} T_{a b} \hat{\tau}^{a} \ell^{b} d^{3} V=\frac{1}{8 \pi G} \int_{\Delta H} \frac{1}{r^{2}} d r d^{2} V=\frac{r_{2}-r_{1}}{2 G} .
$$


The gravitational flux, of course, vanishes because of spherical symmetry.

Remark: In the above discussion, we restricted ourselves to dynamical horizons whose cross-sections are spherically symmetric. It is natural to ask if the space-time admits other, non-spherical dynamical horizons. Surprisingly, this question is not easy to analyze because very little is known about non-spherical marginally trapped surfaces even in the Schwarzschild space-time. However, we will show that, within the $v=$ const surfaces, there is no marginally trapped 2-surface which lies entirely outside the surface $r=2 M(v)$ considered here. It would be interesting to know if the space-time admits other dynamical horizons and, if so, whether the one discussed here is Hayward's trapping boundary [15], discussed in section II B.

Let us then look for a closed 2-surface $S^{\prime}$ given by $r=2 G M(v)-h(\theta, \phi)$ which is marginally trapped. By construction, it lies on the constant $v$ slices. Let $\tilde{\ell}^{a}$ and $\tilde{n}^{a}$ be null normals to this 2-surface. One can show that $\tilde{n}^{a}=\bar{n}^{a}$ but $\tilde{\ell}^{a} \neq \bar{\ell}^{a}$. It can also be shown that the ingoing null expansion to is still $\Theta_{(\tilde{n})}=-4 / r$ while the outgoing expansion becomes

$$
\Theta_{(\tilde{\ell})}=-\frac{h}{r^{2}}+\frac{\Delta_{0} h}{r^{2}}+\frac{\left|D_{0} h\right|^{2}}{r^{3}}
$$

where $\Delta_{0}$ and $D_{0}$ are respectively the standard Laplacian and derivative operator on the unit 2-sphere in $(\theta, \phi)$ coordinates. By setting $\Theta_{(\tilde{\ell})}=0$ we obtain the following partial differential equation for $h(\theta, \phi)$ :

$$
\Delta_{0} h-h=-\frac{\left|D_{0} h\right|^{2}}{r}
$$

As expected, $h=0$ is clearly a solution; but is it the unique solution? Integrate both sides of eq. (A11) using the standard unit 2-sphere volume element and obtain the inequality $\oint_{S^{\prime}} h>0$. This tells us that we cannot have solutions to eq. (A11) with $h$ everywhere negative. In other words, we cannot have marginally trapped surfaces which lie completely outside $r=2 G M(v)$. Of course, the analysis is incomplete because it does not preclude surfaces which lie only partially outside $r=2 G M$ nor surfaces which do not lie on the $v=$ const slices; these issues are currently under investigation.

\section{Inclusion of the cosmological constant}

The example presented in the last sub-section can be generalized to include a cosmological constant $\Lambda$. For definiteness, we restrict ourselves the $\Lambda>0$ case. The Vaidya metric in the presence of a cosmological constant is

$$
g_{a b}=-\left(1-\frac{2 G M(v)}{r}-\frac{\Lambda r^{2}}{3}\right) \nabla_{a} v \nabla_{b} v+2 \nabla_{(a} v \nabla_{b)} r+r^{2}\left(\nabla_{a} \theta \nabla_{b} \theta+\sin ^{2} \theta \nabla_{a} \phi \nabla_{b} \phi\right) .
$$

As before, $M(v)$ is a non-decreasing function of $v$. When $M$ is a constant, this is just the usual Schwarzschild-de Sitter solution. As we shall see below, this solution admits a black hole horizon only if the inequality $9 \Lambda(G M)^{2} \leq 1$ is satisfied. In the remainder of this section we shall always assume that $G M$ never exceeds the value $(9 \Lambda)^{-1 / 2}$. The Einstein tensor for the metric given above is

$$
G_{a b}=-\Lambda g_{a b}+\frac{2 G \dot{M}}{r^{2}} \nabla_{a} v \nabla_{b} v
$$


As before, the stress energy tensor $T_{a b}$ is

$$
T_{a b}=\frac{\dot{M}(v)}{4 \pi r^{2}} \nabla_{a} v \nabla_{b} v
$$

and $\dot{M} \geq 0$ is required in order to satisfy the null energy condition. In this case, there are two horizons: the usual black hole horizon and also a cosmological horizon which are given by the solutions of the equation

$$
f(v, r):=1-\frac{2 G M(v)}{r}-\frac{\Lambda r^{2}}{3}=0 .
$$

This is a cubic equation in $r$ and when $0<9 \Lambda(G M)^{2}<1$, it admits precisely two real and positive solutions given by

$$
r_{c}=\frac{2}{\sqrt{\Lambda}} \cos \left(\frac{\pi-\alpha}{3}\right) \quad \text { and } \quad r_{b}=\frac{2}{\sqrt{\Lambda}} \cos \left(\frac{\pi+\alpha}{3}\right)
$$

where $\alpha=\cos ^{-1}\left(\sqrt{9 \Lambda(G M)^{2}}\right)$. The black hole horizon is located at $r_{b}$ and the cosmological horizon at $r_{c}$. In general, $r_{b} \leq r_{c}$ and when $9 \Lambda(G M)^{2}=1$, the two horizons coincide: $r_{b}=r_{c}=\sqrt{3 / \Lambda}$. When $9 \Lambda(G M)^{2}>1$, then equation (A15) does not admit any real positive solutions. Assuming that $M$ is an increasing function of $v$, it is easy to see that $r_{b}$ increases with time and $r_{c}$ decreases with time and both horizons merge in the limit $G M^{2} \rightarrow 1 / 9 \Lambda$

The derivatives of $f(v, r)$ are

$$
f^{\prime}=\frac{\partial f}{\partial r}=\frac{2 G M}{r^{2}}-\frac{2 \Lambda r}{3} \quad \text { and } \quad \dot{f}=\frac{\partial f}{\partial v}=-\frac{2 G \dot{M}}{r}<0
$$

At the horizons, when $f=0$, the expression for $f^{\prime}$ simplifies to

$$
\left.f^{\prime}\right|_{f=0}=\frac{1-\Lambda r^{2}}{r} .
$$

The derivative $f^{\prime}$ is positive at the black hole horizon and negative at the cosmological horizon.

As before, let us look for all possible spherically symmetric marginally trapped surfaces. The null normals to the $r=$ constant, $v=$ constant surfaces can be taken to be

$$
\bar{\ell}^{a}=\left(\frac{\partial}{\partial v}\right)^{a}+\frac{f}{2}\left(\frac{\partial}{\partial r}\right)^{a} \quad \text { and } \quad \bar{n}^{a}=-2\left(\frac{\partial}{\partial r}\right)^{a} .
$$

The expansions of these null normals are

$$
\Theta_{(\bar{\ell})}=\frac{f}{r} \quad \text { and } \quad \Theta_{(\bar{n})}=-\frac{4}{r} .
$$

Thus we see that $\Theta_{(\bar{n})}$ is always negative and $\Theta_{(\bar{\ell})}$ vanishes precisely at the two horizons. Furthermore,

$$
\left.\mathcal{L}_{\bar{n}} \Theta_{(\bar{\ell})}\right|_{f=0}=-\frac{2 f^{\prime}}{r}
$$


which tells us that $\mathcal{L}_{\bar{n}} \Theta_{(\bar{\ell})}<0$ at $r=r_{b}$ and $>0$ at $r=r_{c}$. Thus, if $\dot{M}>0$, the surface $r=r_{b}$ is space-like and is a dynamical horizon while $r=r_{c}$ is time-like and is, in fact, a time-like dynamical horizon as discussed in appendix B.

In the remainder of this section, we focus only on the black hole horizon. All the remaining equations in this sub-section are valid only at $r=r_{b}$. The unit normal to the horizon is

$$
\hat{\tau}_{a}=\frac{1}{\sqrt{\left|2 \dot{f} f^{\prime}\right|}}\left[\dot{f} \nabla_{a} v+f^{\prime} \nabla_{a} r\right] \quad \text { and } \quad \hat{\tau}^{a}=\frac{1}{\sqrt{\left|2 \dot{f} f^{\prime}\right|}}\left[f^{\prime}\left(\frac{\partial}{\partial v}\right)^{a}+\dot{f}\left(\frac{\partial}{\partial r}\right)^{a}\right] \text {. }
$$

The constant $r$ surfaces are the preferred cross sections of the horizon and the unit space-like normal $\hat{r}^{a}$ to these cross sections is

$$
\hat{r}_{a}=\frac{1}{\sqrt{\left|2 \dot{f} f^{\prime}\right|}}\left[-\dot{f} \nabla_{a} v+f^{\prime} \nabla_{a} r\right] \quad \text { and } \quad \hat{r}^{a}=\frac{1}{\sqrt{\left|2 \dot{f} f^{\prime}\right|}}\left[f^{\prime}\left(\frac{\partial}{\partial v}\right)^{a}-\dot{f}\left(\frac{\partial}{\partial r}\right)^{a}\right]
$$

The properly rescaled null normals are

$$
\ell^{a}=\frac{2\left|f^{\prime}\right|}{\sqrt{\left|2 \dot{f} f^{\prime}\right|}}\left(\frac{\partial}{\partial v}\right)^{a} \quad \text { and } \quad n^{a}=\frac{2 \dot{f}}{\sqrt{\left|2 \dot{f} f^{\prime}\right|}}\left(\frac{\partial}{\partial r}\right)^{a}
$$

The lapse function corresponding to the radial coordinate $r$, which in this case is also the area radius, is given by

$$
N_{r}=\left|\frac{\dot{f}}{2 f^{\prime}}\right|^{1 / 2}=\sqrt{\frac{G \dot{M}}{\left|1-\Lambda r^{2}\right|}}
$$

and thus the properly rescaled vector field corresponding to the radial coordinate $r$ is $t_{o}^{a}=$ $N_{r} \ell^{a}=(\partial / \partial v)^{a}$.

To calculate the flux law, let us first compute $T_{a b} \hat{\tau}^{a} \ell^{b}$ :

$$
T_{a b} \hat{\tau}^{a} \ell^{b}=\left(\frac{2 \dot{M}}{r^{2}}\right) \frac{\left|f^{\prime}\right|}{\sqrt{\left|2 \dot{f} f^{\prime}\right|}} \cdot \frac{2\left|f^{\prime}\right|}{\sqrt{\left|2 \dot{f} f^{\prime}\right|}}=\frac{f^{\prime}}{r}=\frac{1-\Lambda r^{2}}{r^{2}} .
$$

Therefore, the matter flux across the dynamical horizon is:

$$
\mathcal{F}_{\text {matter }}^{(r)}=\int_{\Delta H} N_{r} T_{a b} \hat{\tau}^{a} \ell^{b} d^{3} V=\frac{1}{8 \pi G} \int_{\Delta H}\left(\frac{1}{r^{2}}-\Lambda\right) d r d^{2} V
$$

and the mass function on the horizon is

$$
E^{t_{0}}(r)=\frac{r}{2 G}-\frac{\Lambda r^{3}}{6 G}=M(v)
$$

whence, as expected, the infinitesimal form of the first law takes the form (5.10). 


\section{APPENDIX B: TIME-LIKE ANALOGS OF DYNAMICAL HORIZONS}

In the analysis presented in this paper, the space-like character of the dynamical horizon played a crucial role. However, as we saw in appendix A 2, the time-like case can occur in cosmological contexts. We do not expect the matter or the gravitational fluxes to be generally positive definite in this case whence, in particular, the topology of cross-sections need not be restricted. However, for simplicity of presentation, we shall consider only the case of spherical topology; the generalization to higher genus cross-sections is obvious.

Definition: A smooth, three-dimensional, time-like sub-manifold $H$ in a space-time is said to be a time-like dynamical horizon if it is foliated by a family of space-like 2-spheres such that on each leaf, the expansion $\theta_{(\ell)}$ of a null normal $\ell^{a}$ vanishes while the expansion $\theta_{(n)}$ of the other null normal $n^{a}$ is strictly negative.

The notation will follow the space-like case as much as possible. The main difference is that $\widehat{r}_{a}$ and $\widehat{\tau}_{a}$ now have different meanings. $\widehat{r}_{a}$ is no longer tangential to $H$, it is instead the unit space-like vector normal to $H$. Similarly, $\widehat{\tau}_{a}$ is the unit time-like vector tangential to $H$ and orthogonal to the cross-sections of $H$. As before, the null normals are

$$
\ell^{a}=\widehat{\tau}^{a}+\widehat{r}^{a} \quad \text { and } \quad n^{a}=\widehat{\tau}^{a}-\widehat{r}^{a} .
$$

As one would expect, the time-like case involves many quantities which are analogues of their space-like counterparts, usually with $\widehat{r}_{a}$ and $\widehat{\tau}_{a}$ interchanged. These quantities will be denoted with primes.

What happens to the area increase law now? Looking at the expressions for the expansions of the null normals, one can easily check that

$$
D_{a} \widehat{\tau}^{a}=\theta_{(\ell)}+\theta_{(n)}<0
$$

This clearly shows that the area of the cross-sections decreases along $\widehat{\tau}^{a}$.

As in the space-like case, the analysis of the flux law will be based on the constraint equations on $H$. In the time-like case, the only difference in the constraint equations is a sign change in the scalar constraint (compare with eqs. (2.4) and (2.5)):

$$
\begin{aligned}
H_{S}^{\prime} & :=-\mathcal{R}+K^{2}-K^{a b} K_{a b}=16 \pi G \bar{T}_{a b} \widehat{r}^{a} \widehat{r}^{b} \\
H_{V}^{\prime a} & :=D_{b}\left(K^{a b}-K q^{a b}\right)=8 \pi G \bar{T}^{b c} \widehat{r}_{c} q^{a}{ }_{b}
\end{aligned}
$$

where, as in the main text, $\bar{T}_{a b}$ is related to the matter stress-energy $T_{a b}$ via $\bar{T}_{a b}=T_{a b}-$ $(1 / 8 \pi G) \Lambda g_{a b}$. Once again, we focus our attention on the energy flux along the vector $\xi_{(t)}^{a}=$ $N_{t} \ell^{a}$ where the lapse function $N_{t}$ is now tied to the choice of a time coordinate $t$ on $H$ by the equation

$$
D_{a} t=N_{t} \widehat{\tau}_{a}
$$

where level surfaces of $t$ are the cross sections of $H$ and we have the same rescaling freedom in the lapse as before. The expression for the matter energy flux along $\xi_{(t)}^{a}$ is now given by

$$
\mathcal{F}_{\text {matter }}^{(t)}:=\int_{\Delta H} \bar{T}_{a b} \widehat{r}^{a} \xi_{(r)}^{b} d^{3} V=\frac{1}{16 \pi G} \int_{\Delta H} N_{t}\left(-\mathcal{R}+K^{2}-K^{a b} K_{a b}+2 \widehat{\tau}_{a} D_{b} P^{a b}\right) d^{3} V
$$

the only difference from the space-like being the different sign of the scalar curvature term. 
Using the Gauss-Codacci equation relating the curvatures of $H$ and $S \subset H$ leads to

$$
-\mathcal{R}=2\left(\mathcal{R}_{a b}-\mathcal{G}_{a b}\right) \widehat{\tau}^{a} \widehat{\tau}^{b}=-\widetilde{\mathcal{R}}+\widetilde{K}^{2}-\widetilde{K}_{a b} \widetilde{K}^{a b}+2 D_{a} \alpha^{\prime a}
$$

where

$$
\alpha^{\prime a}=\widehat{\tau}^{b} D_{b} \widehat{\tau}^{a}-\widehat{\tau}^{a} D_{b} \widehat{\tau}^{b} .
$$

The momentum constraint is unchanged and so the flux becomes

$\mathcal{F}_{\text {matter }}^{(t)}:=\frac{1}{16 \pi G} \int_{\Delta H} N_{t}\left(-\widetilde{\mathcal{R}}+\widetilde{K}^{2}-\widetilde{K}^{a b} \widetilde{K}_{a b}+K^{2}-K_{a b} K^{a b}-2 P^{a b} D_{a} \widehat{\tau}_{b}+2 D_{a} \gamma^{\prime a}\right) d^{3} V$,

where $\gamma^{\prime a}=\alpha^{\prime a}+\beta^{\prime a}$ and $\beta^{\prime a}=K^{a b} \widehat{\tau}_{b}-K \widehat{\tau}^{a}$.

The decomposition of the extrinsic curvatures $K_{a b}$ and $\widetilde{K}_{a b}$ proceeds exactly as before. However, the analogues of eqs. (3.17) and (3.16) now have some negative signs:

$$
\widehat{\tau}^{a} D_{a} \widehat{\tau}_{b}=-\frac{\widetilde{D}_{b} N_{t}}{N_{t}} \quad \text { and } \quad \gamma^{a}=\widetilde{q}_{b}^{a} \gamma^{b}=\widehat{\tau}^{b} D_{b} \widehat{\tau}^{a}-\widetilde{W}^{a} .
$$

The simplified flux equation then becomes

$$
\mathcal{F}_{\text {matter }}^{(t)}=\frac{1}{16 \pi G} \int_{\Delta H} N_{t}\left(-\widetilde{\mathcal{R}}-|\sigma|^{2}+2\left|\zeta^{\prime}\right|^{2}\right) d^{3} V
$$

where $\zeta^{\prime a}$ is the analog of $\zeta^{a}$ :

$$
\zeta^{\prime a}=\widetilde{q}^{a b} \widehat{\tau}^{c} \nabla_{c} \ell_{b}
$$

The area balance law now reads

$$
\left(\frac{R_{2}}{2 G}-\frac{R_{1}}{2 G}\right)=-\int_{\Delta H} \bar{T}_{a b} \widehat{r}^{a} \xi_{(t)}^{b} d^{3} V-\frac{1}{16 \pi G} \int_{\Delta H} N_{t}\left\{|\sigma|^{2}-2\left|\zeta^{\prime}\right|^{2}\right\} d^{3} V,
$$

where $\widehat{r}^{a}$ and $\zeta^{a}$ are space-like. Therefore, even though the area decreases monotonically, neither the matter term nor the geometrical terms on the right side have definite signs. This is yet another illustration of the fact that the form of the area balance law (3.23) for dynamical horizons is very special.

[1] M. Heusler, Black Hole Uniqueness Theorems (Cambridge University Press: Cambridge) (1996).

[2] L. Mysak and G. Szekeres, Behavior of the Schwarzschild Singularity in Superimposed Gravitational Fields Can.J.Phys. 44617 (1966);

F.J. Ernst, Black holes in a magnetic universe J. Math. Phys. 17 No.1, $54-56$ (1976);

R. Geroch and J.B. Hartle, Distorted Black Holes J. Math. Phys. 23(4) 680 (1982)

S. Fairhurst and B. Krishnan, Distorted black holes with charge Int. J. Mod. Phys. D10 691-710 (2001) (Preprint gr-qc/0010088)

[3] A. Ashtekar, C. Beetle and S. Fairhurst, Mechanics of Isolated Horizons, Class.Quant.Grav. 17 253-298 (2000) (Preprint: gr-qc/9907068) 
[4] A. Ashtekar, C. Beetle, O. Dreyer, S. Fairhurst, B. Krishnan, J. Lewandowski and J. Wiśniewski, Generic Isolated Horizons and their Applications Phys. Rev. Lett. 853564 (2000) (Preprint gr-qc/0006006)

[5] A. Ashtekar, C. Beetle and J. Lewandowski, Geometry of Generic Isolated Horizons, Class. Quantum Grav. 191195 (2002) (Preprint gr-qc/0111067)

[6] A. Ashtekar, S. Fairhurst and B. Krishnan, Isolated Horizons: Hamiltonian Evolution and the First Law Phys. Rev. D62 104025 (2000) (Preprint gr-qc/0005083)

[7] A. Ashtekar, C. Beetle and J. Lewandowski, Mechanics of Rotating Isolated Horizons Phys. Rev. D64 044016 (2001) (Preprint gr-qc/0103026)

[8] S. W. Hawking, In Black Holes, Les Houches lectures (1972), Edited by C. DeWitt and B.S. DeWitt (North Holland, Amsterdam)

[9] S. W. Hawking and G.F.R. Ellis, Large scale structure of spacetime (Cambridge Uinversity Press, Cambridge, 1972).

[10] H. Bondi, M.G.J. Van der Burg and A.W.K. Metzner, Gravitational waves in general relativity VII: Waves from axi-symmetric isolated systems Proc. Roy. Soc. Lond. A269, 21 (1962)

[11] A. Ashtekar and M. Streubel, Symplective geometry of radiative fields at null infinity Proc. R. Soc (London) A376, 585-607 (1981).

[12] R.M. Wald and A. Zoupas, General Definition of "Conserved Quantities" in General Relativity and Other Theories of Gravity Phys. Rev. D61 084027 (2000) (Preprint: gr-qc/9911095)

[13] R. Penrose, Naked Singularities, N. Y. Acad. Sci. 224, 125-134 (1973)

[14] G. Huisken and T. Ilmanen, The inverse mean curvature flow and the Riemannian Penrose inequality J. Diff. Geo. 59, 353-437-267 (2001)

H. Bray, Proof of the Riemannian Penrose inequality using positive mass theorem J. Diff. Geo. 59, 177 (2001)

[15] S. Hayward, General laws of black hole dynamics Phys. Rev. D49, 6467-6474 (1994) (Preprint: gr-qc/9303006)

S. Hayward, Spin-Coefficient Form of the New Laws of Black-Hole Dynamics, Class. Quant. Grav. 11, 3025-3036 (1994) (Pre-print gr-qc/9406033)

[16] A. Ashtekar and B. Krishnan, Dynamical horizons: energy, angular momentum, fluxes and balance laws Phys. Rev. Lett. 89, 261101 (2002) (Preprint: gr-qc/0207080)

[17] A. Ashtekar, How Black holes grow, Proceedings of the international conference on Mathematical Physics, General Relativity and Cosmology, held in honor of Professor Jerzy Plebanski in Mexico city, in September of 2002 (Preprint gr-qc/0306115)

[18] A. Ashtekar, S. Hayward and B. Krishnan (in preparation)

[19] Shapiro and Teukolsky,Collisions of relativistic clusters and the formation of black holes, Phys. Rev. D45, 2739-2750 (1992)

[20] L. Lehner, N.T. Bishop, R. Gomez, B. Szilagyi and J. Winicour, Exact solutions for the intrinsic geometry of black hole coalescence Phys. Rev. D60, 044005 (1999) (Preprint: gr-qc/9809034)

S. Husa and J. Winicour, The asymmetric merger of black holes Phys. Rev. D60, 084019 (1999) (Preprint: gr-qc/9905039)

[21] E. Witten, A new proof of the positive energy theorem, Commun. Math. Phys., 80, 381-402 (1981)

[22] I. Booth and S. Fairhurst, Hamiltonian framework for dynamical horizons (2002) (in preparation)

[23] J. Hartle and S.W. Hawking, Energy and angular momentum flow in to a black hole Commun. 
Math. Phys. 27, 283-290 (1972)

[24] S. Chandrasekhar, The Mathematical Theory of Black Holes, Oxford University Press (1983)

[25] R. M. Wald R M, Quantum Field Theory in Curved Spacetime and Black Hole Thermodynamics, Chicago: University of Chicago Press (1994)

[26] J. Lewandowski and T. Pawloski, Geometric Characterizations of the Kerr isolated horizon, Int. J. Mod. Phys. D11 739-746 (2001) (Preprint: gr-qc/0101008)

[27] I. Booth and S. Fairhurst, The first law for slowly evolving horizons, Preprint: gr-qc/0307087 (2003)

[28] A. Ashtekar, J. Baez, A. Corichi and K. Krasnov, Quantum geometry and black hole entropy Phys. Rev. Lett. 80904 (1998) (Preprint gr-qc/9710007)

A. Ashtekar, A. Corichi and K. Krasnov, Isolated horizons: the classical phase space Adv. Theor. Math. Phys. 3419 (1999) (Preprint gr-qc/9905089)

A. Ashtekar, J. Baez, K. Krasnov, Quantum geometry of isolated horizons and black hole entropy Adv. Theo. Math. Phys 41 (2000) (Preprint gr-qc/0005126)

[29] A. Ashtekar, http://www.phys.psu.edu/events/index.html?event_id=517 (2003)

[30] P. Anninos, D. Bernstein, S. Brandt, D. Hobill, E. Seidel, L.Smarr, Dynamics of Black Hole Apparent Horizons, Phys. Rev. D 50 3801-3819 (1994)

P. Anninos, D. Bernstein, S. Brandt, D. Hobill, E. Seidel, L.Smarr, Oscillating Apparent Horizons in Numerically Generated Spacetimes, Aust. J. Phys. 48 1027-1043 (1995). 